\title{
Scaling pulser output parameters for standard and dry brick configurations
}

\author{
R. V. Shapovalov $\odot,{ }^{1, *}$ R. B. Spielman, ${ }^{2}$ P.-A. Gourdain, ${ }^{2}$ and R. D. McBride® ${ }^{1}$ \\ ${ }^{1}$ University of Michigan, Department of Nuclear Engineering and Radiological Sciences, \\ Ann Arbor, Michigan 48109, USA \\ ${ }^{2}$ University of Rochester, Laboratory for Laser Energetics, Rochester, New York 14623, USA
}

(Received 30 June 2020; accepted 24 September 2020; published 22 October 2020; corrected 25 February 2021)

\begin{abstract}
The building block of the linear transformer driver pulser, known as a brick, can be thought of as the smallest, independent unit from which the entire pulser is built. In a "standard" brick configuration, two capacitors and a spark-gap switch are connected in series and are oil insulated. Such a brick could be assembled, for example, from two 80-nF, 100-kV capacitors and a 200-kV spark-gap switch. A single brick in this configuration is capable of generating a current pulse with up to $50 \mathrm{kA}$ of peak current and a rise time on the order of $100 \mathrm{~ns}$, after the capacitors have been discharged at a combined output voltage of $200 \mathrm{kV}$ (twice the bipolar charge voltage). By contrast, in a "dry" brick configuration, the two capacitors are placed in parallel and a multigap, multichannel "ball" switch follows them in series. The dry brick is epoxy insulated, and its slightly different configuration allows it to have a peak output current of up to $100 \mathrm{kA}$, after being discharged at a total output voltage of $100 \mathrm{kV}$ (equal to the unipolar charge voltage). In this paper, we present how these two different brick topologies, either oil insulated (standard) or epoxy insulated (dry), affect a pulser's output parameters, where the pulser has been constructed by connecting the outputs of $N$ bricks together in parallel. We find that in many cases, the use of a dry brick configuration results in more current being delivered to the load. We also find that if the time to peak current is not a critical parameter for the experiment, then the lower charging voltage across the switch and the absence of insulating oil make the dry brick an excellent alternative to the standard brick, especially for smaller research laboratories with limited maintenance staff.
\end{abstract}

DOI: $10.1103 /$ PhysRevAccelBeams.23.100401

\section{INTRODUCTION}

The linear transformer driver (LTD) was first introduced in 1997 [1] as an alternative to the Arkad'ev-Marx generator. In this first LTD, one low-inductance capacitor and one multichannel, multigap switch were connected to form a unit, and two such units were connected in parallel to directly drive a discharge circuit without additional cables or long transmission lines. In modern LTDs, the single unit, called a brick, is comprised of two capacitors and a spark-gap switch connected in series, and many bricks are connected in parallel inside the single LTD cavity [2-11]. This brick topology is not unique though; around the same time, a different brick configuration was introduced [12-14], with two capacitors connected in parallel (instead of in series) followed by a low-inductance, multigap, multichannel ball switch [12] (instead of a single-channel spark-gap switch, such as the Russian HCEI switch

\footnotetext{
*shaproma@umich.edu
}

Published by the American Physical Society under the terms of the Creative Commons Attribution 4.0 International license. Further distribution of this work must maintain attribution to the author(s) and the published article's title, journal citation, and DOI.
[15-17], originally used in all standard LTDs, or the more recent L3Harris switch, model 50264). This type of brick has been widely used to build large LTDs [18,19] and small pulsers [20], and it has been proposed for use in future experiments [21]. In this paper, we refer to the oil-insulated type of brick (two capacitors and a spark-gap switch discharged in series) as a standard brick, and to the epoxyinsulated type of brick (two capacitors in parallel and a multigap, multichannel ball switch in series) as a dry brick.

A thorough overview of LTD technologies has been presented by Kim [22,23], describing both standard and dry brick LTDs, and by Mazarakis [5], describing the LTD development at Sandia National Laboratories. In this paper, we further examine how two different brick topologies (standard and dry brick topologies) affect a pulser's output parameters, where the pulser has been constructed by connecting the outputs of $N$ bricks together in parallel. For a system based on either type of brick, when the pulser drives a transmission line and a matched load that both have small inductances relative to the inductance of the pulser, the peak output current into the load scales linearly with the number of bricks $N$, and the time to peak current is independent of $N$. In this case, the dry-brick pulser outperforms the standard-brick pulser-i.e., relative to the 

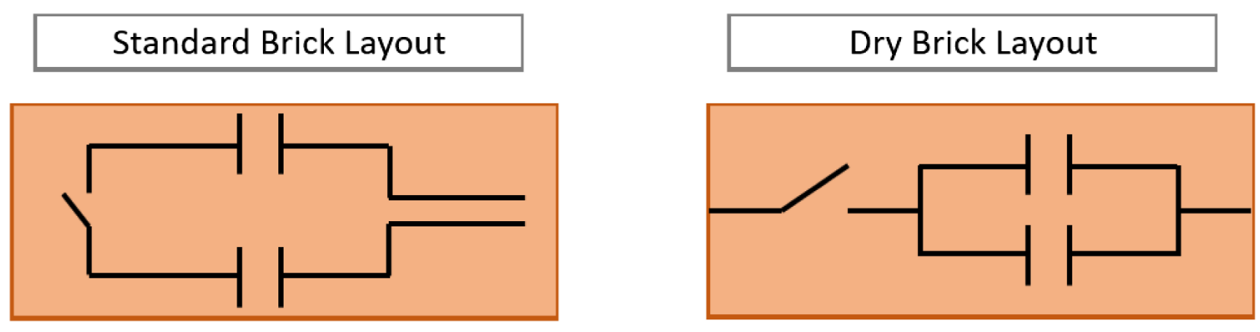

FIG. 1. Schematic layouts for "standard" and "dry" brick configurations.

standard-brick pulser, the dry-brick pulser generates a larger peak current. By contrast, when the inductance of the transmission line and/or the load is large compared to the inductance of the pulser, both the peak current and the rise time scale as $\sqrt{ } N$; additionally, the peak current becomes independent of the type of brick for large $N$. If the time to peak current is not a critical parameter for the experiment, then the lower charging voltage across the switch and the absence of insulating oil make the dry brick an excellent alternative to the standard brick, especially for smaller research laboratories with limited maintenance staff. Most of the examples presented in this paper are given for a brick comprised of $80-\mathrm{nF}, 100-\mathrm{kV}$ capacitors, but the general scaling analyses provided in this paper can be easily applied to other capacitor values as well.

The two different brick topologies, standard and dry, are schematically presented in Fig. 1. If we assume that a single capacitor can handle no more than a 50-kA peak current (which is typical for modern $80-\mathrm{nF}, 100-\mathrm{kV}$ capacitors ${ }^{1}$ ), then the peak current for the standard brick will be limited to $50 \mathrm{kA}$, while the dry brick can operate at a larger 100-kA peak current. The other difference is in the brick's charging voltages; the standard brick can be bipolar charged up to $\pm 100 \mathrm{kV}$ on the spark-gap side of capacitors, and, after the spark gap is triggered, its effective peak output voltage becomes $200 \mathrm{kV}$; by contrast, the dry brick is unipolar charged to $100 \mathrm{kV}$, and thus the output voltage is $100 \mathrm{kV}$. The difference of bipolar charging versus unipolar charging means that, in our example, prior to the switch closing, the standard brick has $200 \mathrm{kV}$ applied across the switch, while the dry brick has only $100 \mathrm{kV}$ applied across the switch. Consequently, the standard brick must be immersed in insulating oil to prevent high-voltage breakdown during normal operation, while the dry brick (as its name suggests) does not require the insulating oil; its components can be potted in solid dielectric material (epoxy, for example), and the entire brick can be operated at normal atmospheric conditions. (The dry brick is often referred to as an "air-insulated brick"). The higher output current and lower charging voltage across the switch, combined with simplified operation and maintenance (absence of insulating oil, bipolar charging systems, and high-pressure gas

\footnotetext{
${ }^{1}$ Note that the most recent GA 35478 capacitors can now handle up to $60 \mathrm{kA}$ per capacitor.
}

systems-i.e., the multigap, multichannel ball switch operates in normal ambient air conditions), makes the dry brick attractive for many pulsed-power applications.

The brick, either standard or dry, can be thought of as the elementary building block from which an entire pulser is constructed. It is then useful to explore how the pulser's output parameters depend on the brick topology. For the purpose of the present paper, we will only be discussing pulsers where all bricks are connected in parallel and, after being fully charged, all bricks are simultaneously triggered into the discharge circuit comprised of a short transmission line (TL) and load. Examples of pulsers where bricks are arranged in parallel are presented in Fig. 2. Both 2(a) and 2(b) represent an LTD type pulser, where bricks are arranged around the perimeter of a cylindrical cavity: the LTD in 2(a) is comprised of standard bricks, while the LTD in 2(b) is comprised of dry bricks. Here, magnetic cores are used for inductive isolation-i.e., to ensure that the current flows in the circuit path that includes the TL and the load rather than the parasitic path around the inside of the metal LTD case. The use of magnetic cores permits one to stack LTD cavities in series to generate higher output voltages. Examples of compact pulsers with very small footprints, where bricks are connected in parallel, are presented in Fig. 2(c) and 2(d). Figure 2(c) is an example of a pulser comprised of two dry bricks which have been arranged in a side-by-side geometry [20], while Fig. 2(d) is an example of a pulser comprised of five standard bricks hung vertically from the bent TL [24].

The remainder of this paper is organized as follows. In Sec. II, we present the basic circuit parameters for the components found in the standard and dry brick configurations. In Sec. III, we present how the system's overall performance scales with the number of bricks used, either standard or dry, assuming that the extra inductance of the TL and load is small compared to the pulser's inherent inductance. Section IV explores the pulser scaling relations when the inductance of the transmission line and load cannot be neglected. In both Secs. III and IV, we are dealing with the so-called "matched" load case, $R=\sqrt{L / C}$, where $\mathrm{R}, \mathrm{L}$ and $\mathrm{C}$ are the total system resistance, inductance, and capacitance, respectfully, and where the system consists of the pulser, the TL, and the load taken together as a whole. The matched load condition is vital for designing a compact high-current pulser [25], where the pulser's output current is maximized, yet the voltage reversal at the brick's 
(a)

LTD with Standard Brick Layout

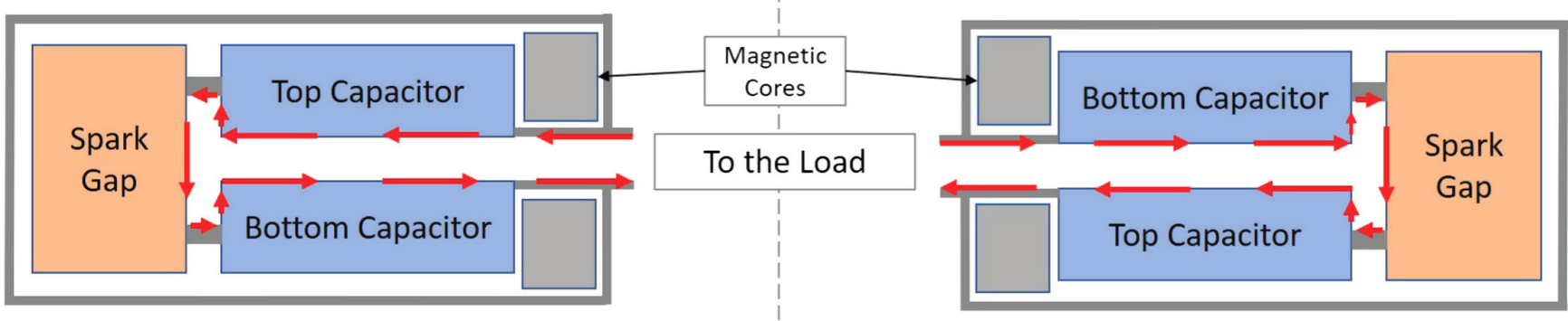

(b) LTD with Dry Brick Layout

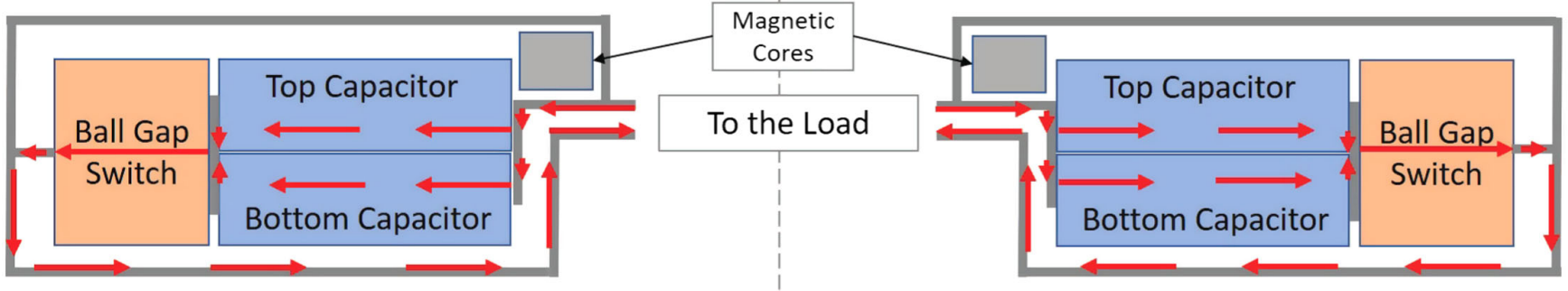

(c)

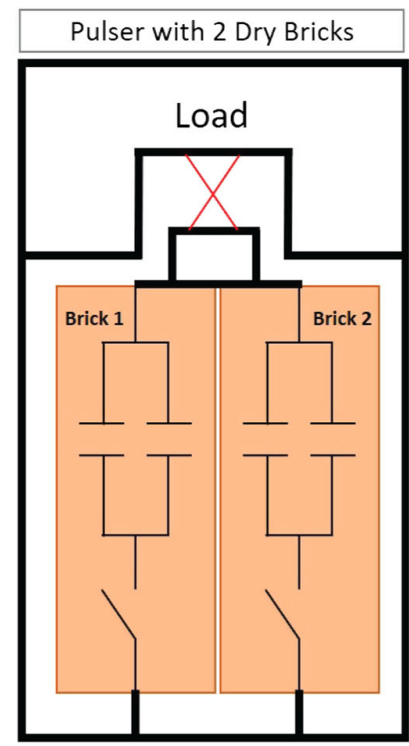

(d)

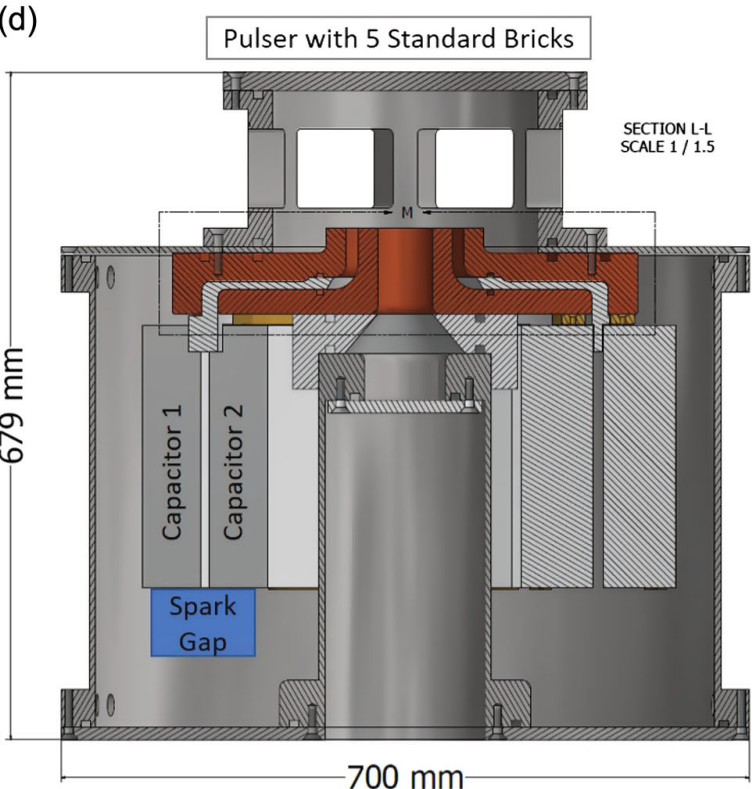

FIG. 2. Pulsers with brick outputs connected in parallel: (a) standard brick LTD; (b) dry brick LTD; (c) dry-brick pulser that uses two bricks placed side by side, with the output TL and load residing above the bricks; and (d) standard-brick pulser that uses five bricks hung vertically, with the output TL and load residing above the bricks. The pulsers in (c) and (d) are examples of compact pulsers with very small footprints.

capacitors remains below the maximum recommended by the capacitor manufacturers (this is usually $20 \%-30 \%$ of the capacitors' maximum charge voltage). In Sec. V, we present a few examples and discuss how an unmatched load affects the pulser's performance. In Sec. VI, we summarize our results and discuss the implications for the scaling relations presented for both types of pulsers.

\section{II. “STANDARD” VERSUS “DRY” BRICK CIRCUIT TOPOLOGY}

The circuit representations for both the standard and dry brick configurations are presented in Fig. 3. Each brick is comprised of two capacitors and a closing switch, but they are arranged differently depending on the type of the brick. In a standard brick configuration (Fig. 3, left), all elements 


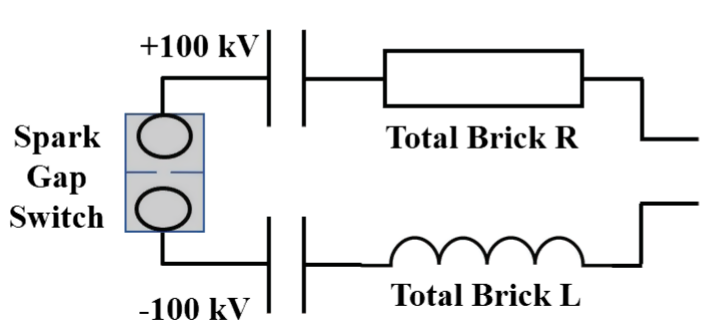

$+100 \mathrm{kV}$

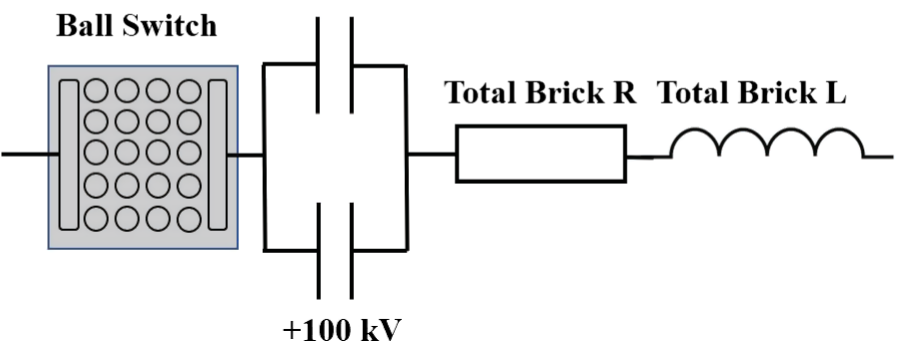

FIG. 3. The standard (left) versus dry (right) brick topology.

are placed in series, with the top and bottom capacitors bridged in the middle by a spark-gap switch. In a dry brick configuration (Fig. 3, right), both capacitors are connected in parallel and are followed by a multigap, multichannel ball switch (ball-gap switch [12]) in series. Due to this difference in brick configuration, the electrical characteristics of standard and dry bricks are fundamentally different.

As can be seen in Fig. 3, with a standard brick configuration, the total brick current will be limited by the maximum allowed current of a single capacitor, but with a dry brick configuration, the total brick current is twice the maximum allowed current of a single capacitor. If we assume that each capacitor can handle a maximum allowed current of 50-kA inside its normal operational limit, then the maximum brick current will be limited to $50 \mathrm{kA}$ for the standard brick, and $100 \mathrm{kA}$ for the dry brick. This fact alone makes a dry brick a very good option for many low-impedance, high-current applications. For example, for a given number of bricks in the system, one can reach a higher load current if dry bricks are used instead of standard bricks. Alternatively, to reach the same load current, a smaller number of bricks is required if dry bricks are used instead of standard bricks. Additionally, as mentioned in the Introduction, the lower charge voltage that appears across the switch for dry bricks $(100 \mathrm{kV})$ allows them to be potted in dielectric material (e.g., epoxy) and to be operated under normal atmospheric conditions without insulating oil. Note that in the standard brick, the charge voltage that appears across the switch is $200 \mathrm{kV}$, due to the $\pm 100-\mathrm{kV}$ bipolar charging used in the standard brick.

It can be seen from Fig. 3 that the capacitance of the dry brick is 4 times greater than the capacitance of the standard brick:

$$
C_{\text {dry }}=4 C_{\text {stand }} \text {. }
$$

For example, for a single capacitor value of $80 \mathrm{nF}$, the capacitance of a standard brick will be $40 \mathrm{nF}$, while the capacitance of a dry brick will be $160 \mathrm{nF}$, which is 4 times greater than the standard brick's capacitance. The total energy stored remains the same.
The brick inductance is not as straightforward to evaluate as the capacitance, since the equivalent single-brick inductance depends not only on contributions from the individual components (i.e., the capacitors and the switch), but also on the packaging geometry of the components within the brick itself and on the packaging geometry of the $N$ bricks within the overall system. For example, referring to the standard brick configuration in Fig. 2(a), note how the area of the current loop depends on the vertical spacing between the two capacitors. Similarly, referring to the dry brick configuration in Fig. 2(b), note how the area of the current loop depends on the vertical spacing between the bottom capacitor and the bottom of the LTD case. For either type of brick, the inductance depends on the length of the capacitors and switch. Additionally, since $N$ bricks are distributed azimuthally around the outer circumference of the LTD cavity, the azimuthal spacing between the bricks can affect the effective inductance value of a single brick (i.e., the mutual inductances between the bricks can become important as the azimuthal spacing between the bricks decreases). Even for the same type of brick and capacitors, the effective single-brick inductance may vary largely. If we ignore the complications associated with brick packaging geometry, then it would be safe to state that, for similar-sized capacitors, the inductance of the dry brick will be less than the inductance of the standard brick:

$$
L_{\text {dry }}<L_{\text {standard }} \text {. }
$$

To further discuss the brick's output parameters, we assume a matched load solution,

$$
R_{\text {system }}=R_{\text {matched }} \equiv \sqrt{L_{\text {system }} / C_{\text {system }}},
$$

where $R_{\text {system }}$ is the total system resistance (which includes the resistance of all the bricks connected in parallel, the $\mathrm{TL}$, and the load), and $\sqrt{L_{\text {system }} / C_{\text {system }}}$ is they system's characteristic impedance. The matched load solution allows one to maximize the peak current delivered to the load while also protecting the capacitors from excessive voltage and current reversals, which can occur if the system is significantly underdamped (i.e., if 
$R_{\text {system }} \ll R_{\text {matched }} \equiv \sqrt{L_{\text {system }} / C_{\text {system }}}$. It is common to consider the matched load solution when designing a highcurrent pulser. For the matched case (2.3), the brick peak current, peak voltage, and time-to-peak current into the matched load can be shown [25] to be equal to

$$
\begin{gathered}
I_{\text {brick, matched }}=\frac{0.55 V_{0}}{R_{\text {brick, matched }}}=\frac{0.55 V_{0}}{\sqrt{L_{\text {brick }} / C_{\text {brick }}}} \\
V_{\text {brick, matched }}=0.55 V_{0} \\
t_{\text {brick, matched }}=1.2 \sqrt{L_{\text {brick }} C_{\text {brick }}} .
\end{gathered}
$$

Considering (2.1) and (2.2), and recalling that the effective charging voltage is $200 \mathrm{kV}$ for the standard brick (due to bipolar charging) and $100 \mathrm{kV}$ for the dry brick (due to unipolar charging), it is easy to see that

$$
\begin{aligned}
& I_{\text {matched, dry }}>I_{\text {matched, stand }} \\
& t_{\text {matched, dry }}>t_{\text {matched, stand }} .
\end{aligned}
$$

The circuit parameters in Eqs. (2.3)-(2.6), as calculated for several standard and dry brick configurations, are presented in Table I. The first part of Table I (rows 1-14) is for standard brick configurations, and the second part (rows 15-18) is for dry brick configurations. $C_{b}$ and $L_{b}$ (columns 4 and 5) are the total brick capacitance and inductance values, and $R_{m}, I_{m}, V_{m}, T_{m}$ (columns 6-9) are the matched values for the bricks, calculated using
Eqs. (2.3)-(2.6). The brick inductance, $L_{b}$, was either directly measured from brick testing $[17,26,27,13]$, or was evaluated from LTD testing [3,6] (20-nF case). The brick inductances $[24,20]$ are from the short-circuit tests, and inductances $[8,6](58-\mathrm{nF}$ case) are estimates from calculations. $I_{\exp }$ (column 10) is the measured peak current into the matched load per single brick that was either directly measured from brick testing [20-22,13] or was evaluated from LTD testing $[4,6]$.

It is interesting to note that the inductance for different bricks varies largely even for the same type of capacitors. As was already discussed, the inductance of the brick depends not only on the internal inductances of the capacitors and switch, but also on a brick's construction; there is always some extra inductance term which depends on the brick's volume, length, inner electrode gap, etc. It also depends on how one defines the brick's boundary or how the brick's inductance has been measured. To make the comparison between standard and dry bricks more straightforward, we assume that both the standard and dry bricks are made of similar sized $80-\mathrm{nF}, 100-\mathrm{kV}$ capacitors. As suggested by (2.2), we would expect the inductance of the dry brick to be less than the inductance of the standard brick. Moreover, by examining the corresponding inductances from Table I, it would be safe to state that the inductance of the standard brick, comprised of two $80-\mathrm{nF}, 100-\mathrm{kV}$ capacitors, is about $180 \mathrm{nH}$, while the inductance of the dry brick, comprised of similar $80-\mathrm{nF}$,

\begin{tabular}{|c|c|c|c|c|c|c|c|c|c|c|}
\hline Brick & Capacitor & Switch & $C_{B} \mathrm{nF}$ & $L_{B} \mathrm{nH}$ & $R_{m} \Omega$ & $I_{m}, \mathrm{kA}$ & $V_{m} \mathrm{kV}$ & $T_{m} \mathrm{~ns}$ & $I_{\text {exp }} \mathrm{kA}$ & Reference \\
\hline Standard & GA35436 $8 \mathrm{nF} / 100 \mathrm{kV}$ & HCEI Tomsk & 4 & 150 & 6.12 & 18 & 110 & 30 & 15 & [17] \\
\hline Standard & GA35425 $40 \mathrm{nF} / 100 \mathrm{kV}$ & HCEI Tomsk & 20 & 254 & 3.56 & 31 & 110 & 86 & 24 & [3] \\
\hline Standard & GA35426 $38 \mathrm{nF} / 100 \mathrm{kV}$ & HCEI Tomsk & 19 & 185 & 3.12 & 35 & 110 & 72 & 33.4 & [26] \\
\hline Standard & GA35426 $38 \mathrm{nF} / 100 \mathrm{kV}$ & Sandia & 19 & 136 & 2.68 & 41 & 110 & 62 & 40 & [26] \\
\hline Standard & GA35426 $38 \mathrm{nF} / 100 \mathrm{kV}$ & Kinetech & 19 & 171 & 3.00 & 37 & 110 & 69 & 37 & [26] \\
\hline \multirow[t]{2}{*}{ Standard } & GA35426 $38 \mathrm{nF} / 100 \mathrm{kV}$ & L3 Large & 19 & 164 & 2.94 & 37 & 110 & 68 & 36 & [26] \\
\hline & GA35426 $38 \mathrm{nF} / 100 \mathrm{kV}$ & L3 Sandia & 20 & 155 & 2.77 & 40 & 110 & 68 & 41.1 & [27] \\
\hline Standard & GA35426 $38 \mathrm{nF} / 100 \mathrm{kV}$ & Kinetech-2 & 20 & 139 & 2.62 & 42 & 110 & 64 & 40.6 & [27] \\
\hline Standard & GA35426 $40 \mathrm{nF} / 100 \mathrm{kV}$ & L3 Sandia & 20 & 182 & 3.02 & 36 & 110 & 73 & 33 & {$[6]$} \\
\hline Standard & $58 \mathrm{nF} / 100 \mathrm{kV}$ & L3 Sandia & 29 & 172 & 2.44 & 45 & 110 & 85 & 43 & {$[6]$} \\
\hline Standard & $40 \mathrm{nF} / 100 \mathrm{kV}$ & HCEI Tomsk & 20 & 240 & 3.46 & 32 & 110 & 84 & $\cdots$ & [28] \\
\hline Standard & CSI $80 \mathrm{nF} / 100 \mathrm{kV}$ & In-house UR & 40 & 176 & 2.10 & 52 & 110 & 102 & $\cdots$ & [24] \\
\hline Standard & Ximai $80 \mathrm{nF} / 100 \mathrm{kV}$ & MGS-I China & 40 & 268 & 2.59 & 42 & 110 & 125 & $\cdots$ & [8] \\
\hline Standard & Ximai $80 \mathrm{nF} / 100 \mathrm{kV}$ & MGS-II China & 40 & 245 & 2.47 & 44 & 110 & 120 & $\cdots$ & [8] \\
\hline Dry & GA35436 $8 \mathrm{nF} / 100 \mathrm{kV}$ & 6-ch 7-gaps & 16 & 50 & 1.77 & 31 & 55 & 34 & 25 & [13] \\
\hline Dry & GA35426 $40 \mathrm{nF} / 100 \mathrm{kV}$ & 6-ch 7-gaps & 80 & 50 & 0.79 & 70 & 55 & 77 & 48 & [13] \\
\hline Dry & GA35465 $140 \mathrm{nF} / 100 \mathrm{kV}$ & 5-ch 7-gaps & 280 & 104 & 0.61 & 90 & 55 & 206 & $\cdots$ & [20] \\
\hline Dry & $150 \mathrm{nF} / 100 \mathrm{kV}$ & 5-ch 7-gaps & 300 & 100 & 0.58 & 95 & 55 & 210 & $\cdots$ & [21] \\
\hline Standard & $80 \mathrm{nF} / 100 \mathrm{kV}$ & Spark gap & 40 & 180 & 2.12 & 52 & 110 & 103 & $\ldots$ & \\
\hline Dry & $80 \mathrm{nF} / 100 \mathrm{kV}$ & Ball switch & 160 & 90 & 0.75 & 73 & 55 & 145 & $\cdots$ & \\
\hline
\end{tabular}
$100-\mathrm{kV}$ capacitors, is about $90 \mathrm{nH}$. The corresponding circuit parameters are presented in the last two rows of Table I.

TABLE I. The matched load circuit parameters for standard and dry bricks. 
As expected from (2.7) and (2.8), the dry brick, in general, outputs a higher peak current into the matched load as compared to the standard brick, but its time to peak is longer. For example, for the $80-\mathrm{nF}$ bricks (rows 19 and 20 in Table I), the peak current into the matched load is $52 \mathrm{kA}$ for the standard bricks and about $73 \mathrm{kA}$ for the dry bricks, which is $40 \%$ larger. It is important to note that for this brick configuration, the peak current for the standard brick is slightly above the limit of what each brick capacitor can withstand, while for the dry brick's configuration, the peak current is sufficiently below the capacitor limit. This is the key reason why the typical standard brick is comprised of capacitors with a capacitance value not greater than $80 \mathrm{nF}$, while the dry brick is normally comprised of larger capacitors, with capacitance values of up to $150 \mathrm{nF}$ (rows 17 and 18 in Table I). The down side of the dry bricks is that their time to peak current will be sufficiently longer than that for the standard bricks-e.g., for bricks with 80-nF capacitors, the time to peak current will be 103 ns for the standard brick configuration and $145 \mathrm{~ns}$ for the dry brick configuration. Note, however, that the rise rate of the current pulse in these systems is similar; it just takes longer for the dry bricks to fully discharge.

The matched resistance for the standard brick is about 3 times larger than the corresponding matched resistance of the dry brick. In this sense, the standard brick is more suitable for driving a higher impedance load, while the dry brick is more applicable for a lower impedance load. For both brick architectures, when the outputs of many bricks are connected together in parallel, the matched resistance for the system decreases, and thus both pulsers become more suitable for driving lower impedance loads (this is further discussed in Secs. III and IV).

The important conclusion here is that the dry brick can output twice as much peak current as the standard brick, with a maximum value of $50 \mathrm{kA}$ for the standard brick and $100 \mathrm{kA}$ for the dry brick. Furthermore, a pulser comprised of dry bricks (rather than standard bricks) will generate a higher current into a matched load. Additionally, as will be shown next, when the inductance of the TL and load is small compared to the pulser's inductance, the pulser's total output current scales directly with the number of bricks $N$. Therefore, the same peak current into the load could be achieved with a smaller number of bricks if dry bricks are used instead of standard bricks. Combined with a lower effective charge voltage (due to unipolar charging), these higher peak currents make the dry-brick pulser a good alternative to the standard-brick pulser.

In the following sections, we discuss how the pulser's output parameters are scaled with the number of bricks. The following assumptions in all circuit simulations have been made.

First, we assumed that for the overall systems that we are evaluating, the transit time of an electromagnetic wave through the system is short compared to the rise time of the current pulse. Therefore, the lumped circuit approximation is valid, and the entire system can be described by a simple RLC model, where $\mathrm{R}, \mathrm{L}$, and $\mathrm{C}$ are the total system resistance, inductance, and capacitance values, respectively, and where the system is comprised of the pulser, the TL, and the load.

Second, the total $\mathrm{R}, \mathrm{L}$ and $\mathrm{C}$ values are assumed to be constant for each brick configuration we consider. One limitation of such a static RLC model is that it overpredicts the peak brick current. For example, as can be seen from Table I, for the standard brick comprised of 8-nF, GA35436 capacitors and HCEI switch, the current amplitude into the matched load calculated using static RLC model is $18 \mathrm{kA}$, while the measured current is $15 \mathrm{kA}$ (16\% difference). For the standard brick comprised of 40-nF, GA35426 capacitors and an L3/Sandia switch, the current amplitude into the matched load calculated using the static RLC model is $36 \mathrm{kA}$, while the measured current is $33 \mathrm{kA}$ (9\% difference). For the dry brick comprised of 40-nF, GA35426 capacitors and a ball switch with six channels and seven gaps, the current amplitude into the matched load calculated using static RLC model is $70 \mathrm{kA}$, while the measured current is $48 \mathrm{kA}$ ( $31 \%$ difference). Such differences are not surprising $[13,17]$, as they account for the energy dissipated in the switch. The total current flowing through a brick can be modeled using the Braginskii [29] switch model, which describes the evolution of the spark gap channel in a dense gas or liquid. In this model, the resistance of the current channel is inverse proportional to the current action integral $C \int_{0}^{t} I^{2 / 3} d t$, where the constant $\mathrm{C}$ is a function of the spark gap tabulated mass density, electrical conductivity, specific heats, and the switch total gap length, and operational pressure.

To estimate the effect of the switch energy losses for the standard and dry bricks, we performed a series of simulations that compare the brick current into the matched load calculated using the static RLC model and using the Braginskii formalism. Simulations have been performed using the pulsed-power circuit code SCREAMER [30]. SCREAMER uses the Martin lossy switch model [31], which has been carefully compared to detailed spark gap experiments and agrees very well with them over a large range of operating conditions. Simulation results are presented in Fig. 4. The left plot corresponds to the standard brick with total $\mathrm{C}$ and $\mathrm{L}$ values of $40 \mathrm{nF}$ and $180 \mathrm{nH}$, respectively (Table I, rows 19), while the right plot is for the dry brick with total $\mathrm{C}$ and $\mathrm{L}$ values of $160 \mathrm{nF}$ and $90 \mathrm{nH}$, respectively (Table I, rows 20). The thick black line presents the static RLC switch model with a matched R value of $2.12 \Omega$ for the standard brick, and $0.75 \Omega$ for the dry brick. For the Martin switch model (thin black lines), the load resistance has been varied until the reversal peak current reached $16 \%$ of the positive peak value that agreed with the static RLC model. The switch used for the standard brick model is the L3 switch [26] that has two 12-mm gaps and operates at 

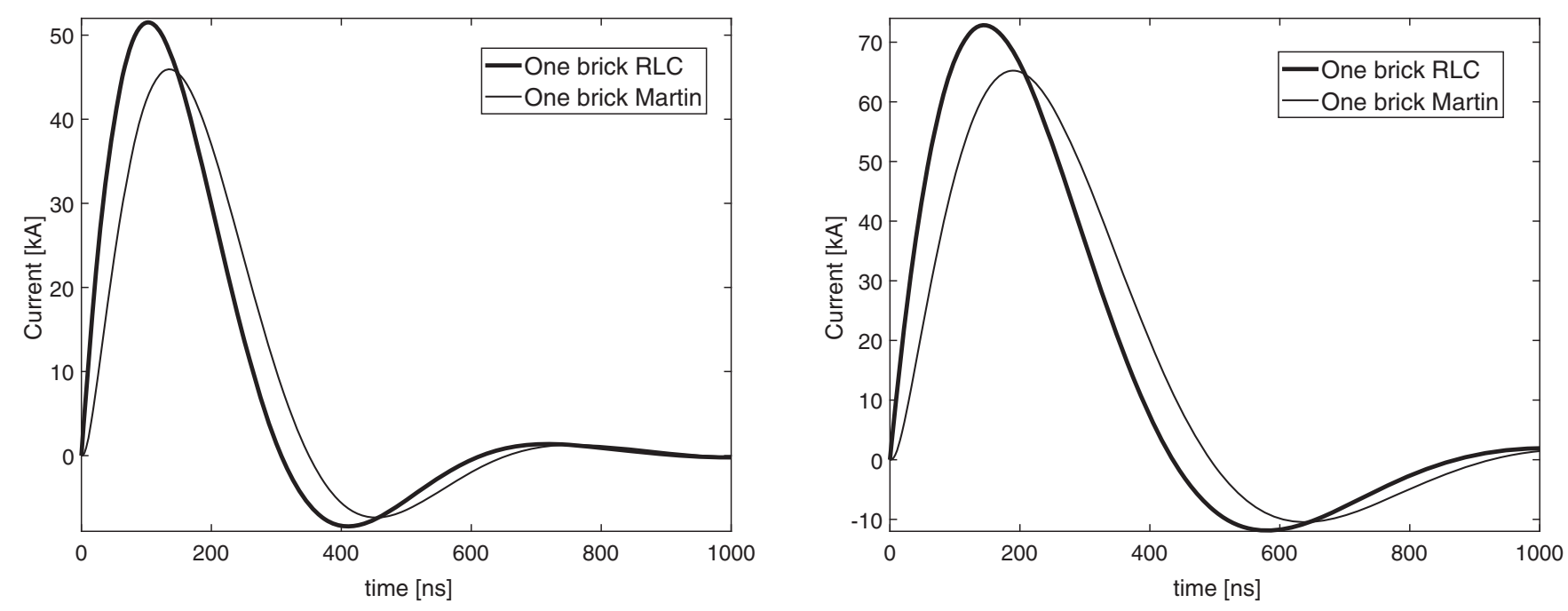

FIG. 4. Simulated output currents into a matched load for a standard brick (left) and a dry brick (right). The thick line is for the static switch model, and the thin line is for the Martin switch model.

72 psia. The switch used for the dry brick model is a ball switch with five channels and seven gaps [20], where the total gap length is $42 \mathrm{~mm}$ per channel and the operating pressure is $1 \mathrm{~atm}$.

In the left plot of Fig. 4 (standard brick), the current amplitude into the matched load, calculated using the static RLC switch model (thick line), is $52 \mathrm{kA}$, while the current amplitude based on the Martin lossy switch model is about $46 \mathrm{kA}$ ( $11 \%$ difference). For the dry brick (right plot in Fig. 4), the static RLC model yields 73 kA peak current, while the Martin lossy switch model predicts $65 \mathrm{kA}$ peak current (also $11 \%$ difference). The calculated reduction in the current amplitude is due to the switch energy losses and are in general agreement with values presented in Table I. The above simulations have been extended for the pulser comprised of 10 and 20 similar bricks. It was found that the total reduction in the brick current is independent of the number of bricks and is about $11 \%$ for both the standard and dry configurations.

To estimate the effect of the reduction in the brick current amplitude, depending on the switch design and its operating conditions, we performed a series of simulations that compare four different cases: the standard brick comprised of (1) HCEI Russian switches, (2) Sandia switches, (3) Kinetech switch, or (4) L3 switches. All four switches are single-channel spark gaps with various parameters described in Ref. [26]. The HCEI Russia switch has six, 6-mm gaps and operates at 57 psia. The Sandia switch has two, 12-mm gaps and operates at $130 \mathrm{psia}$. The Kinetech switch has two, 5-mm gaps and operates at 242 psia. The L3 switch has two, 12-mm gaps and operates at 72 psia. Simulation results are presented in Fig. 5. All cases correspond to the standard brick configuration with a total $\mathrm{C}$ of $40 \mathrm{nF}$. The brick inductance has two parts. The first part is $90 \mathrm{nH}$ and accounts for the total inductance of the capacitors, the load, and all of the connecting components; this inductance is fixed for all four cases. The second part is the switch inductance, which is $115 \mathrm{nH}$ for the HCEI switch, $66 \mathrm{nH}$ for the Sandia switch, $100 \mathrm{nH}$ for the Kinetech switch, or $93 \mathrm{nH}$ for the L3 switch [26]. In all four cases, the load resistance has been varied until the $16 \%$ current reversal has been achieved, which corresponds to a matched load condition. As can be seen in Fig. 5, different switch configurations result in different current amplitudes: the peak currents are $42 \mathrm{kA}$ for the HCEI switch (black solid line), $48 \mathrm{kA}$ for the Sandia switch (black dashed line), $47 \mathrm{kA}$ for the Kinetech switch (black dotted line), and $46 \mathrm{kA}$ for the L3 switch (black dash-dotted line). The red line corresponds to the static RLC brick model presented in Fig. 4 and is plotted here for comparison. Simulated peak

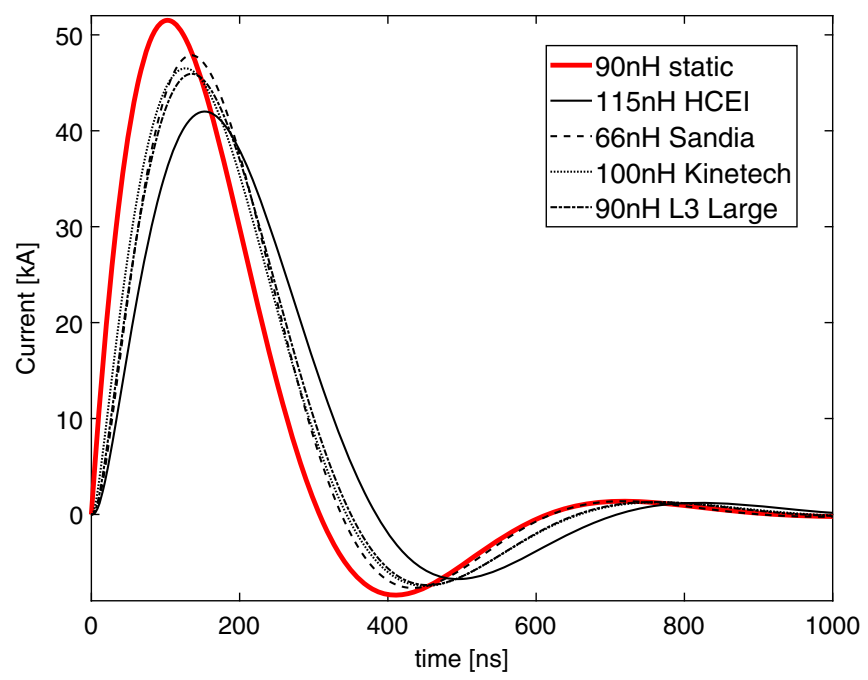

FIG. 5. Simulated output currents into matched load conditions for a standard brick assuming four different switch configurations. 
current amplitudes are in good relative agreement with measured values presented in Table I, rows 3-6.

Throughout this paper, when comparing the standard brick configuration with the dry brick configuration, we have made use of the static RLC model for simplicity. In general, the total switch energy loss depends on many switch specific parameters (as shown in Fig. 5) and on the parameters of the entire circuit (based on the data in Table I). These additional loss factors should be taken into account when comparing simulation results with experimental measurements; however, these additional loss factors should not impact the primary conclusion of our study, which was to compare the standard brick configuration with the dry brick configuration in a relative sense, while holding all other loss factors constant from one configuration to the next. For example, we note that the additional loss factor in going from a static resistance model to the lossy Martin switch model is approximately the same for each switch (see Figs. 4 and 5). Thus, the inclusion or exclusion of the Martin switch model does not significantly impact the conclusions of our study because the relative differences between the standard brick configuration and the dry brick configuration remain the same regardless of the switch model used.

\section{SCALING THE PULSER'S OUTPUT PARAMETERS WHEN $L_{\text {extra }} \ll L_{\text {pulser }}$}

We first considered how the pulser's output parameters scale with the number of bricks, $N$, when the inductance of the TL and the load can be neglected. Because the brick outputs are connected in parallel, the pulser's total capacitance and inductance values (both for the standard and dry brick topologies), scale exactly with the number of bricks as follows:

$$
\begin{gathered}
C_{\text {pulser }}=C_{\text {brick }} N \\
L_{\text {pulser }}=L_{\text {brick }} / N .
\end{gathered}
$$

Then, with the matched load condition satisfied, the matched resistance, peak current, and time to peak current scale with the number of bricks as follows:

$$
\begin{aligned}
R_{\text {system, matched }} & =\sqrt{L_{\text {system }} / C_{\text {system }}}=\sqrt{\frac{L_{\text {brick }} / N}{C_{\text {brick }} N}} \\
& =\frac{R_{\text {brick, matched }}}{N} \\
I_{\text {system, matched }} & =\frac{0.55 V_{0}}{R_{\text {matched }}}=\frac{0.55 V_{0}}{R_{\text {brick, matched }} / N}=I_{\text {brick, matched }} N \\
t_{\text {system, matched }} & =1.2 \sqrt{\left(C_{\text {brick }} N\right)\left(L_{\text {brick }} / N\right)}=t_{\text {brick, matched }} .
\end{aligned}
$$

As can be seen in (3.3)-(3.5), when the extra inductance of the TL and the load can be neglected, the system's matched load resistance (3.3) scales inversely with the number of bricks, $N$; the pulser's peak current (3.4) scales directly with $N$; and the time to peak current (3.5) becomes independent of $N$. The scaling of the system's output parameters with $N$ (3.1)-(3.5) are presented in Table II, where the brick parameters used correspond to those presented in rows 19 and 20 of Table I (e.g., 80-nF, 100-kV capacitors).

As evidenced by Table II, for the chosen $80-\mathrm{nF}, 100-\mathrm{kV}$ capacitor, the peak current for the pulser comprised of dry bricks is always 1.4 times greater than the peak current of the pulser comprised of standard bricks. For example, for the small pulsers comprised of only four bricks, the peak output currents are about $210 \mathrm{kA}$ for the standard-brick configuration and $290 \mathrm{kA}$ for the dry-brick configuration. Likewise, for the larger pulsers comprised of 40 bricks, the peak output currents are about 2,100 kA for the standard

\begin{tabular}{|c|c|c|c|c|c|c|c|c|c|c|c|c|c|}
\hline \multirow[b]{2}{*}{$\mathrm{N}$ of Bricks } & \multicolumn{2}{|c|}{$C_{\text {total }}[\mathrm{nF}]$} & \multicolumn{2}{|c|}{$L_{\text {pulser }}[\mathrm{nH}]$} & \multirow[b]{2}{*}{$L_{\text {extra }}[\mathrm{nH}]$} & \multicolumn{2}{|c|}{$R_{\text {matched }}[\Omega]$} & \multicolumn{2}{|c|}{$I_{\text {peak }}[\mathrm{kA}]$} & \multicolumn{2}{|c|}{$V_{\text {peak }}[\mathrm{kV}]$} & \multicolumn{2}{|c|}{$t_{\text {peak }}[\mathrm{ns}]$} \\
\hline & Standard & Dry & Standard & Dry & & Standard & Dry & Standard & Dry & Standard & Dry & Standard & Dry \\
\hline 1 & 40 & 160 & 180 & 90 & 0 & 2.12 & 0.75 & 52 & 73 & 110 & 55 & 103 & 145 \\
\hline 2 & 80 & 320 & 90 & 45 & 0 & 1.06 & 0.38 & 104 & 147 & 110 & 55 & 103 & 145 \\
\hline 3 & 120 & 480 & 60 & 30 & 0 & 0.71 & 0.25 & 156 & 220 & 110 & 55 & 103 & 145 \\
\hline 4 & 160 & 640 & 45 & 23 & 0 & 0.53 & 0.19 & 207 & 293 & 110 & 55 & 103 & 145 \\
\hline 5 & 200 & 800 & 36 & 18 & 0 & 0.42 & 0.15 & 259 & 367 & 110 & 55 & 103 & 145 \\
\hline 10 & 400 & 1600 & 18 & 9 & 0 & 0.21 & 0.08 & 519 & 733 & 110 & 55 & 103 & 145 \\
\hline 20 & 800 & 3200 & 9 & 5 & 0 & 0.11 & 0.04 & 1037 & 1467 & 110 & 55 & 103 & 145 \\
\hline 40 & 1600 & 6400 & 5 & 2 & 0 & 0.05 & 0.02 & 2074 & 2933 & 110 & 55 & 103 & 145 \\
\hline
\end{tabular}
brick configuration and 2,900 kA for the dry-brick configuration. As expected, the times to peak current are independent of $N$ and are equal to $103 \mathrm{~ns}$ for the standardbrick pulser and $145 \mathrm{~ns}$ for the dry-brick pulser.

TABLE II. Scaling of system output parameters with $N$ when $L_{\text {extra }} \ll L_{\text {pulser }}$. 
The scaling relations presented in (3.1)-(3.5) allow one to easily estimate how many bricks are needed for the desired output current. For example, for a 500-kA peak currentassuming bricks with $80-\mathrm{nF}, 100-\mathrm{kV}$ capacitors-we would need ten standard bricks or seven dry bricks. We must point out that the scaling relations (3.1)-(3.5) only work when the extra inductance in the system (due to the TL and the load) is small compared to the pulser's internal inductance. As evident from Table II, when a pulser is comprised of a small number of bricks, its internal inductance is relatively large, and the above-mentioned condition is easier to satisfy. For a pulser with many bricks in parallel, the total internal inductance becomes small, and thus for the scaling relations (3.1)-(3.5) to be valid, both the inductance of the TL and the load must be small. An example of a configuration with a low extra inductance is a parallel plate TL with a strip-line load, which are often used in pulsed-power-driven material properties experiments [32].

When the combined inductance of the TL and the load is no longer small compared to the pulser's internal inductance, the scaling of the system's output parameters must be modified according to the description provided in the following section.

\section{SCALING THE PULSER'S OUTPUT PARAMETERS WHEN $\boldsymbol{L}_{\text {extra }}>\boldsymbol{L}_{\text {pulser }}$}

In this section, we consider how the system's output parameters scale with the number of bricks, $N$, when the inductance of the TL and the load cannot be neglected. In this case, the system's total inductance becomes the sum of two terms: the pulser's internal inductance, $L_{\text {pulser }}$, which according to (3.2) scales inversely with the number of bricks, and the system's extra inductance, $L_{\text {extra }}$, which equals the total inductance of the TL and the load and is independent of the number of bricks:

$$
L_{\text {system }}=L_{\text {pulser }}+L_{\text {extra }}=L_{\text {brick }} / N+L_{\text {extra }} .
$$

Then, the system's matched resistance becomes

$R_{\text {system, matched }}=\sqrt{L_{\text {system }} / C_{\text {system }}}=\sqrt{\frac{\left(L_{\text {brick }} / N+L_{\text {extra }}\right)}{C_{\text {brick }} N}}$

which can be shown to be equal to

$$
R_{\text {system, matched }}=\frac{R_{\text {brick, matched }}}{N} \sqrt{\left(1+\frac{L_{\text {extra }}}{L_{\text {pulser }}}\right)} .
$$

Note that when $L_{\text {extra }} \ll L_{\text {pulser }}$, (4.3) reduces to (3.3). When $L_{\text {extra }}$ cannot be neglected, the system's total resistance is inversely proportional to $\sqrt{N}$ :

$$
R_{\text {system, matched }}=\frac{1}{\sqrt{N}} \sqrt{L_{\text {extra }} / C_{\text {brick }}} .
$$

Substituting (4.3) into the expression for the matched peak current (2.4), we find that

$$
\begin{aligned}
I_{\text {system, matched }} & =\frac{0.55 V_{0}}{R_{\text {system, matched }}} \\
& =\frac{0.55 V_{0}}{R_{\text {brick, matched }} / N}\left(1+\frac{L_{\text {extra }}}{L_{\text {pulser }}}\right)^{-\frac{1}{2}} \\
& =I_{\text {brick, matched }} N\left(1+N \frac{L_{\text {extra }}}{L_{\text {brick }}}\right)^{-\frac{1}{2}} .
\end{aligned}
$$

For a large $N$, where $N \cdot L_{\text {extra }} / L_{\text {brick }} \gg 1$, the peak current becomes proportional to $\sqrt{N}$ :

$$
I_{\text {system, matched }}=I_{\text {brick, matched }} \sqrt{L_{\text {brick }} / L_{\text {extra }}} \sqrt{N} \text {. }
$$

In (4.6), it is interesting to note that the factor in front of the $\sqrt{N}$ is independent of the type of brick. For example, for the bricks described by rows 19 and 20 of Table I, and for a system with an extra inductance of $6 \mathrm{nH}$, this factor equals $285 \mathrm{kA}$ for the standard brick configuration and $283 \mathrm{kA}$ for the dry brick configuration. So, with a large $N$, the peak output current into the matched load becomes nearly independent of the type of brick used.

For this case of $L_{\text {extra }}>L_{\text {pulser }}$, the time to peak current depends on $N$ :

$t_{\text {system, matched }}=1.2 \sqrt{\left(L_{\text {brick }} / N+L_{\text {extra }}\right)\left(C_{\text {brick }} N\right)}$,

which can be shown to be equal to

$$
t_{\text {system, matched }}=t_{\text {brick, matched }} \sqrt{\left(1+N \frac{L_{\text {extra }}}{L_{\text {brick }}}\right)} \text {. }
$$

Note that when $L_{\text {extra }} \ll L_{\text {pulser }}=L_{\text {brick }} / N$, (4.8) reduces to (2.6), and the time to peak current becomes independent of $N$. Also note that for $L_{\text {extra }} \gg L_{\text {pulser }}=$ $L_{\text {brick }} / N$, (4.8) can be approximated by

$$
t_{\text {system, matched }}=t_{\text {brick, matched }} \sqrt{L_{\text {extra }} / L_{\text {brick }}} \sqrt{N} .
$$

The factor in front of the $\sqrt{N}$ is different for standard and dry bricks. For the bricks that are described in rows 19 and 20 of Table I, and a system with an extra inductance of $6 \mathrm{nH}$, this factor is $19 \mathrm{~ns}$ for the standard brick configuration and $37 \mathrm{~ns}$ for the dry brick configuration.

For this case of $L_{\text {extra }}>L_{\text {pulser }}$, the scaling of the system's output parameters for both the standard and dry brick configurations are presented in Table III, where the bricks are described in rows 19 and 20 of Table I 
TABLE III. Scaling of system output parameters with $N$ when $L_{\text {extra }}>L_{\text {pulser }}$.

\begin{tabular}{|c|c|c|c|c|c|c|c|c|c|c|c|c|c|}
\hline \multirow[b]{2}{*}{$\mathrm{N}$ of bricks } & \multicolumn{2}{|c|}{$C_{\text {total }}[\mathrm{nF}]$} & \multicolumn{2}{|c|}{$L_{\text {pulser }}[\mathrm{nH}]$} & \multirow[b]{2}{*}{$L_{\text {extra }}[\mathrm{nH}]$} & \multicolumn{2}{|c|}{$R_{\text {matched }}[\Omega]$} & \multicolumn{2}{|c|}{$I_{\text {peak }}[\mathrm{kA}]$} & \multicolumn{2}{|c|}{$V_{\text {peak }}[\mathrm{kV}]$} & \multicolumn{2}{|c|}{$t_{\text {peak }}[\mathrm{ns}]$} \\
\hline & Standard & Dry & Standard & Dry & & Standard & Dry & Standard & Dry & Standard & Dry & Standard & Dry \\
\hline 1 & 40 & 160 & 180 & 90 & 6 & 2.16 & 0.77 & 51 & 71 & 110 & 55 & 104 & 150 \\
\hline 2 & 80 & 320 & 90 & 45 & 6 & 1.10 & 0.40 & 100 & 138 & 110 & 55 & 106 & 155 \\
\hline 3 & 120 & 480 & 60 & 30 & 6 & 0.74 & 0.27 & 148 & 201 & 110 & 55 & 108 & 159 \\
\hline 4 & 160 & 640 & 45 & 23 & 6 & 0.56 & 0.21 & 195 & 261 & 110 & 55 & 109 & 163 \\
\hline 5 & 200 & 800 & 36 & 18 & 6 & 0.46 & 0.17 & 240 & 318 & 110 & 55 & 111 & 168 \\
\hline 10 & 400 & 1600 & 18 & 9 & 6 & 0.24 & 0.10 & 449 & 568 & 110 & 55 & 119 & 187 \\
\hline 20 & 800 & 3200 & 9 & 5 & 6 & 0.14 & 0.06 & 803 & 960 & 110 & 55 & 133 & 222 \\
\hline 40 & 1600 & 6400 & 5 & 2 & 6 & 0.08 & 0.04 & 1358 & 1532 & 110 & 55 & 157 & 278 \\
\hline
\end{tabular}

(e.g., 80-nF, 100-kV capacitors), and where the system contains $6-\mathrm{nH}$ of extra inductance [11,20,24].

As is evident from Table III, when $N$ is small, $I_{\text {peak }}$ is roughly proportional to $N$, and the dry-brick pulser outperforms the standard-brick pulser by a factor of 1.4 in $I_{\text {peak }}$. For large $N, I_{\text {peak }}$ becomes proportional to $\sqrt{N}$, and the differences in $I_{\text {peak }}$ between the standard and dry-brick pulsers becomes less profound; this agrees with (4.6) for the reasons discussed above. In an opposite sense, $t_{\text {peak }}$ begins to differ more between the standard and dry brick cases as $N$ increases: for small $N$, the difference is about $50 \mathrm{~ns}$, while for large $N$, the difference is as much as $120 \mathrm{~ns}$.

As is evidenced by (4.6) and (4.9), for any type of brick architecture, extra inductance in the system will worsen the pulser's performance: as we increase the extra inductance, the system's peak current will drop, and the system's time to peak current will increase. In Fig. 6, plots of $I_{\text {peak }}$ as a function of $N$ are presented for both the standard and dry brick configurations, and for cases where the system includes extra inductances of 6,12 , and $20 \mathrm{nH}$. As can be seen in Fig. 6, as $L_{\text {extra }}$ increases, the difference in $I_{\text {peak }}$

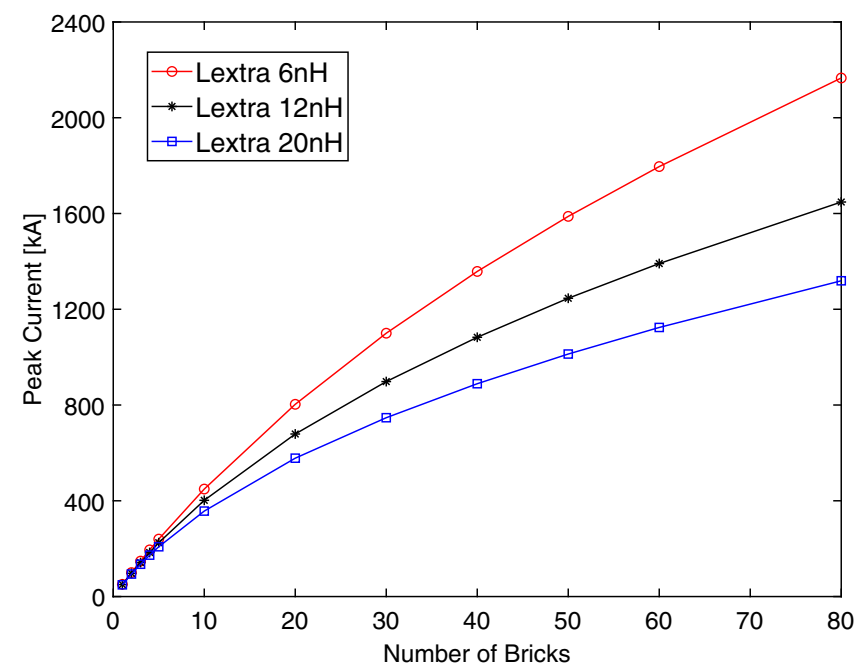

between the standard and dry brick configurations becomes less notable. For example, with $L_{\text {extra }}=20 \mathrm{nH}$ and $N=80$, the $I_{\text {peak }}$ values for the standard and dry-brick pulsers are almost identical: 1,320 kA for the standard brick configuration and $1,350 \mathrm{kA}$ for the dry brick configuration.

To summarize, when $L_{\text {extra }} \ll L_{\text {pulser }}, I_{\text {peak }} \propto N$. This is usually the case for a small pulser (a pulser with a small $N$ ), which often means the pulser's internal inductance is still relatively large. This can also be the case for a large pulser (a pulser with a large $N$ ), as long as the TL and the load have a combined inductance that is very small (an example is a parallel plate TL terminated with a strip-line load). In this case of $L_{\text {extra }} \ll L_{\text {pulser }}$, a pulser comprised of dry bricks is more suitable for higher current applications: using the same number of bricks, it provides a higher output current, or alternatively, to achieve the same output current, the dry-brick pulser requires less bricks total (and does this without the use of insulating oil). Conversely, when the $L_{\text {extra }}$ becomes large enough, $I_{\text {peak }} \propto \sqrt{N}$. In this case, for large $N, I_{\text {peak }}$ for both the standard and dry-brick pulsers becomes almost identical, while $t_{\text {peak }}$ becomes significantly

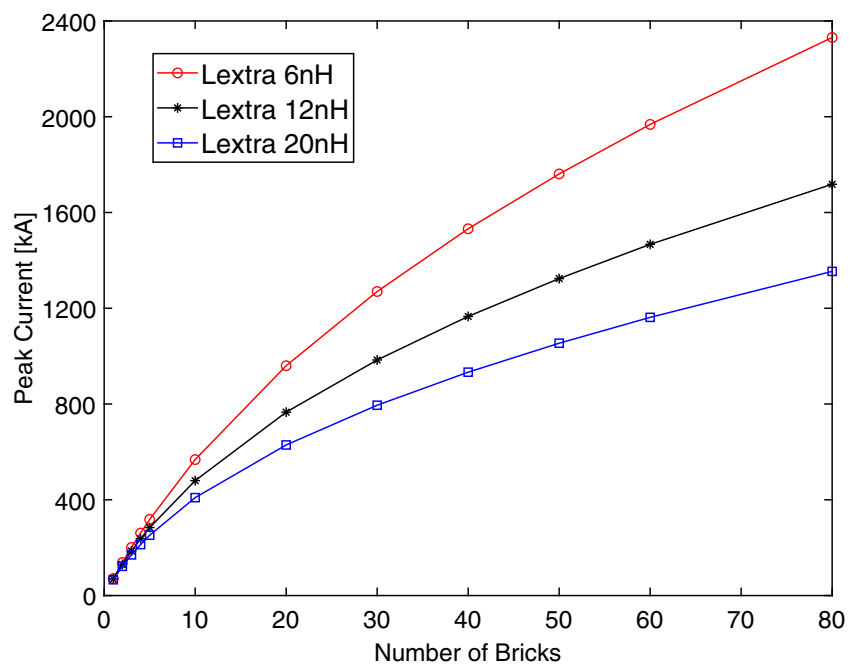

FIG. 6. Scaling of $I_{\text {peak }}$ with $\mathrm{N}$ for the standard brick configuration (left) and the dry brick configuration (right) and for cases with $L_{\text {extra }}=6,12$, and $20 \mathrm{nH}$. The load is always matched. 
larger for the dry brick case. If $I_{\text {peak }}$ for low-impedance loads is the important pulser parameter, then a pulser comprised of dry bricks is a good alternative to the standard-brick pulser - the dry bricks operate under lower effective charging voltages (e.g., $100 \mathrm{kV}$ due to unipolar charging versus $200 \mathrm{kV}$ due to bipolar charging), and, as a result, a dry-brick pulser will be easier to maintain and operate. If having a short $t_{\text {peak }}$ is important, then the standard-brick pulser will outperform the dry-brick pulser.

Up to this point, all of our discussion was based on the matched load solution, which is an important case that allows one to maximize the pulser's output current while keeping the voltage and current reversals below the operational limits of the capacitors. However, it is not always possible to satisfy the matched load condition for a given pulser-load combination. In the next section, we consider several practical examples and discuss how the dry-brick pulser performs relative to the standard-brick pulser under different load conditions.

\section{COMPARISON OF PULSERS WITH MATCHED, INDUCTIVE, AND X-PINCH LOADS}

Here we simulate dry-brick discharges and compare them to standard-brick discharges for three different load scenarios: (1) the matched load $R=\sqrt{L / C}$, (2) a 20-nH inductive load, and (3) an x-pinch load with a 10-nH inductance and a $0.24-\Omega$ resistance. We use the brick parameters given in rows 19 and 20 of Table I-i.e., the parameters for the bricks with $80-\mathrm{nF}, 100-\mathrm{kV}$ capacitors, and thus the standard brick's discharge parameters are $200 \mathrm{kV}, 40 \mathrm{nF}$ and $180 \mathrm{nH}$, while the dry brick's discharge parameters are $100 \mathrm{kV}, 160 \mathrm{nF}$ and $90 \mathrm{nH}$. We use the SCREAMER software code, an open-source pulsed-power circuit tool initially developed at Sandia National Laboratories [30] and currently supported by Spielman

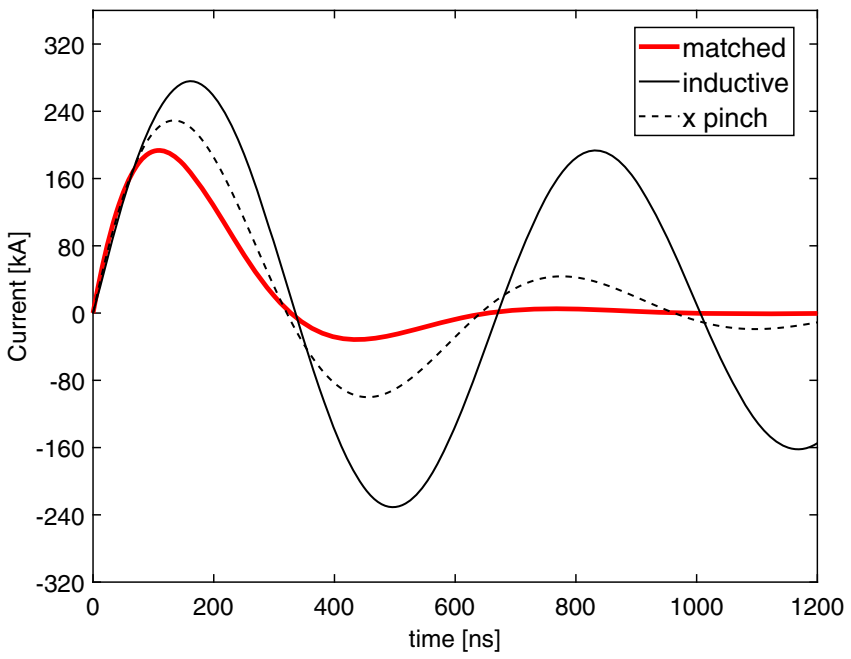

$[33,34]$. Though the circuit simulations performed here are very elementary and can be done with any circuit simulation program, we prefer to use SCREAMER as it allows one to readily implement the many pulsed-power models available in SCREAMER (e.g., the Martin spark-gap switch model, a gas puff load model, etc.) at any point in the future.

\section{A. Small pulser comprised of four bricks}

Let us first consider a small pulser comprised of only four bricks, in either a standard or dry brick configuration. We assume a small 6-nH TL and a load that is either matched, inductive, or x-pinch. The matched load is calculated as follows. First, we set the system's total resistance to $R=\sqrt{L / C}$. Next, we set the load resistance to be the difference between $R$ and the pulser's internal resistance $R_{\text {internal }}=R_{\text {brick }} / N$. We assume that the resistance of the standard brick is $0.3 \Omega$, while the resistance of the dry brick is $0.1 \Omega$, and we neglect the resistance of the TL. The calculated matched load resistances are $0.49 \Omega$ for the standard brick system and $0.19 \Omega$ for the dry brick system.

The simulated output currents into the three different loads are presented in Fig. 7. For the matched load cases (red lines), the $I_{\text {peak }}$ and $t_{\text {peak }}$ values are $190 \mathrm{kA}$ and $110 \mathrm{~ns}$ for the standard-brick pulser and $260 \mathrm{kA}$ and $160 \mathrm{~ns}$ for the dry-brick pulser. These are in very good agreement with the corresponding values from Table III, which were estimated for the matched load case using (3.4) and (3.5). For the purely inductive load (black solid lines), the circuit is nearly undamped, and both pulsers result in a largely oscillating current with a peak value of about $275 \mathrm{kA}$ for the standardbrick pulser and about $340 \mathrm{kA}$ for the dry-brick pulser. For an x-pinch load, it is important to achieve a $1-\mathrm{kA} / \mathrm{ns}$ current rate of rise [35], and both pulsers satisfy these

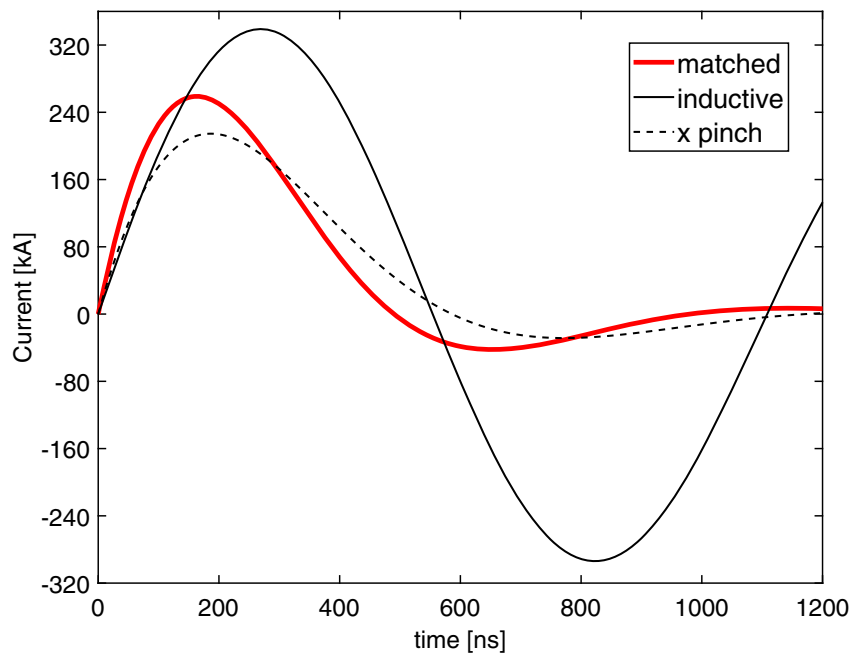

FIG. 7. Simulated output currents into matched, inductive, and x-pinch loads for a standard-brick pulser (left) and a dry-brick pulser (right). Both systems use four bricks and include a 6-nH TL. 

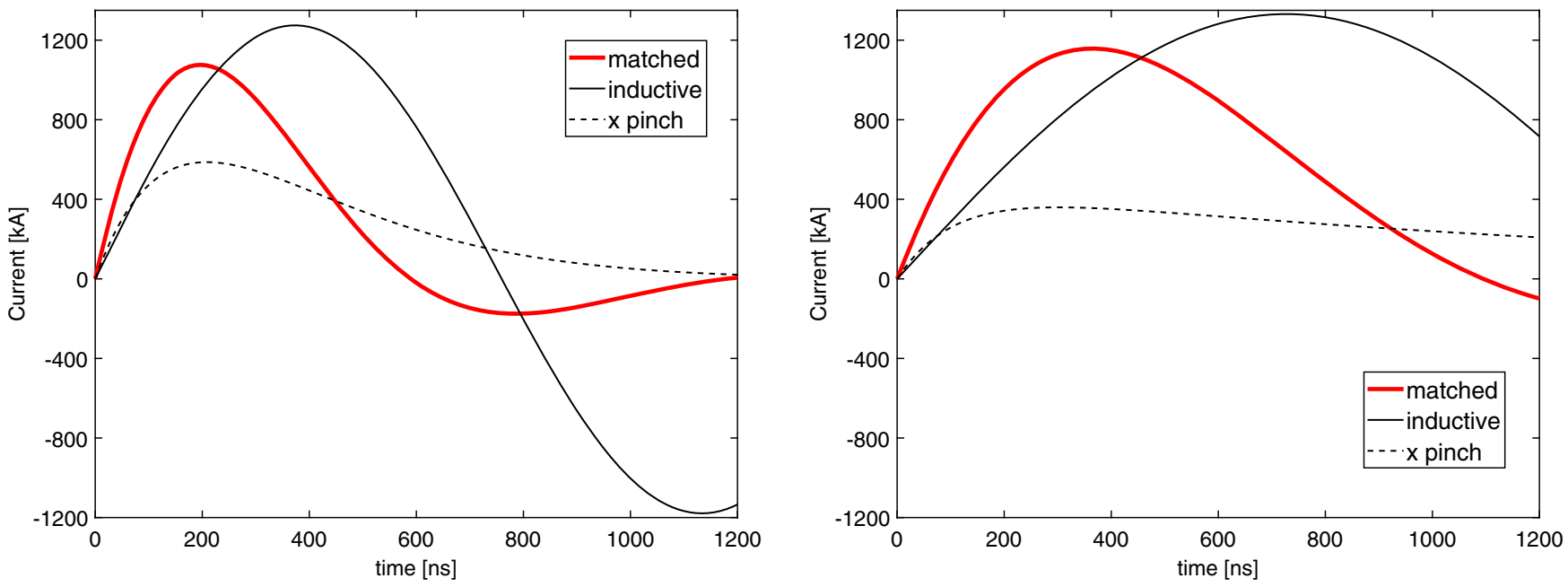

FIG. 8. Simulated output currents into matched, inductive, and x-pinch loads for a 40-brick standard pulser (left) and a 40-brick dry pulser (right). Both systems include a 12-nH TL.

parameters. However, the standard-brick pulser outperforms the dry-brick pulser in $d I / d t$ : the $d I / d t$ is $1.7 \mathrm{kA} / \mathrm{ns}$ for the standard-brick pulser and $1.2 \mathrm{kA} / \mathrm{ns}$ for the dry-brick pulser. It is also interesting to note that for the standard pulser, the $\mathrm{x}$-pinch load results in an increase in the peak output current as compared to the matched load (an increase from $190 \mathrm{kA}$ up to $230 \mathrm{kA}$ ), while for the dry pulser, the x-pinch load results in the drop in the peak current (a decrease from $260 \mathrm{kA}$ down to $215 \mathrm{kA}$ ). This can be explained by the fact that the matched load resistance for the standard-brick pulser is larger than the corresponding $\mathrm{x}$-pinch resistance value $(0.49 \Omega$ vs $0.24 \Omega)$, while for the dry-brick pulser it is smaller $(0.19 \Omega$ vs $0.24 \Omega)$.

\section{B. Large pulser comprised of $\mathbf{4 0}$ bricks}

We next consider a larger pulser comprised of 40 bricks total. The brick discharge parameters are the same as those used in the previous section $(200 \mathrm{kV}, 40 \mathrm{nF}$, and $180 \mathrm{nH}$ for the standard brick, and $100 \mathrm{kV}, 160 \mathrm{nF}$ and $90 \mathrm{nH}$ for the dry brick), and the inductance of the TL is assumed to be either $12 \mathrm{nH}$ (typical for a large LTD TL) or $0.3 \mathrm{nH}$ (typical for a parallel plate TL). The calculated load resistances are $94 \mathrm{~m} \Omega$ for the standard-brick pulser and $45 \mathrm{~m} \Omega$ for the drybrick pulser (for the 12-nH TL case with a matched load) and are 47 and $17 \mathrm{~m} \Omega$ (for the 0.3-nH TL case with a matched load). The parameters of the inductive and $\mathrm{x}$-pinch loads are assumed to be the same as in the previous section. The simulation results for the 40-brick pulser with a 12-nH TL and with a 0.3-nH TL are presented in Figs. 8 and 9, respectively.

As expected for cases where the inductance of the TL is large compared to the pulser's internal inductance, the pulser's peak output current into the matched load becomes independent of the type of bricks used (Fig. 8, red lines). Indeed, the corresponding $I_{\text {peak }}$ is $1,080 \mathrm{kA}$ for the standard-brick pulser and 1,160 kA for the dry-brick pulser. The difference comes in the timing performance: the $t_{\text {peak }}$ for the standard pulser is about $200 \mathrm{~ns}$, while for the dry pulser, it is $360 \mathrm{~ns}$.

The inductive load (Fig. 8, black solid lines) results in an increase in the $I_{\text {peak }}(1,270 \mathrm{kA}$ for the standard-brick pulser and 1,330 kA for the dry-brick pulser) and an increase in $t_{\text {peak }}(370 \mathrm{~ns}$ for the standard-brick pulser and $730 \mathrm{~ns}$ for the dry-brick pulser). Additionally, the circuit becomes largely oscillating (severely underdamped).

For the x-pinch load (Fig. 8, black dashed lines), we observe a significant drop in the pulsers' performances: $I_{\text {peak }}$ drops to $590 \mathrm{kA}$ for the standard-brick pulser and $360 \mathrm{kA}$ for the dry-brick pulser. This can be explained by the fact that when many bricks are connected in parallel, both pulsers have characteristic impedances (i.e., driver impedances) that are small compared to the $x$-pinch resistance, and therefore the entire circuit with the x-pinch load becomes highly resistive and overdamped. It is also evident that the standard pulser outperforms the dry pulser for this case of an x-pinch load: the corresponding $d I / d t$ is $2.8 \mathrm{kA} / \mathrm{ns}$ for the standard pulser and $1.2 \mathrm{kA} / \mathrm{ns}$ for the dry pulser.

For pulsers with a very low-inductance TL (Fig. 9), we expect $I_{\text {peak }}$ into a matched load to be proportional to $N$, and $t_{\text {peak }}$ to be independent of $N$ [see Eqs. (3.4) and (3.5)]. For pulsers with $N=40$ discharging into a matched load (Fig. 9, red lines), the simulated $I_{\text {peak }}$ and $t_{\text {peak }}$ are $1,990 \mathrm{kA}$ and $105 \mathrm{~ns}$ for the standard pulser, and 2,730 kA and $155 \mathrm{~ns}$ for the dry pulser. These results are in good agreement with the expected values from Table II. For an inductive load (Fig. 9, black solid lines), we observe drops in $I_{\text {peak }}$ and increases in the $t_{\text {peak }}$, as expected for an increase in the system's total inductance. The x-pinch load (Fig. 9, black dashed lines) produces even larger drops in $I_{\text {peak }}$, and the 

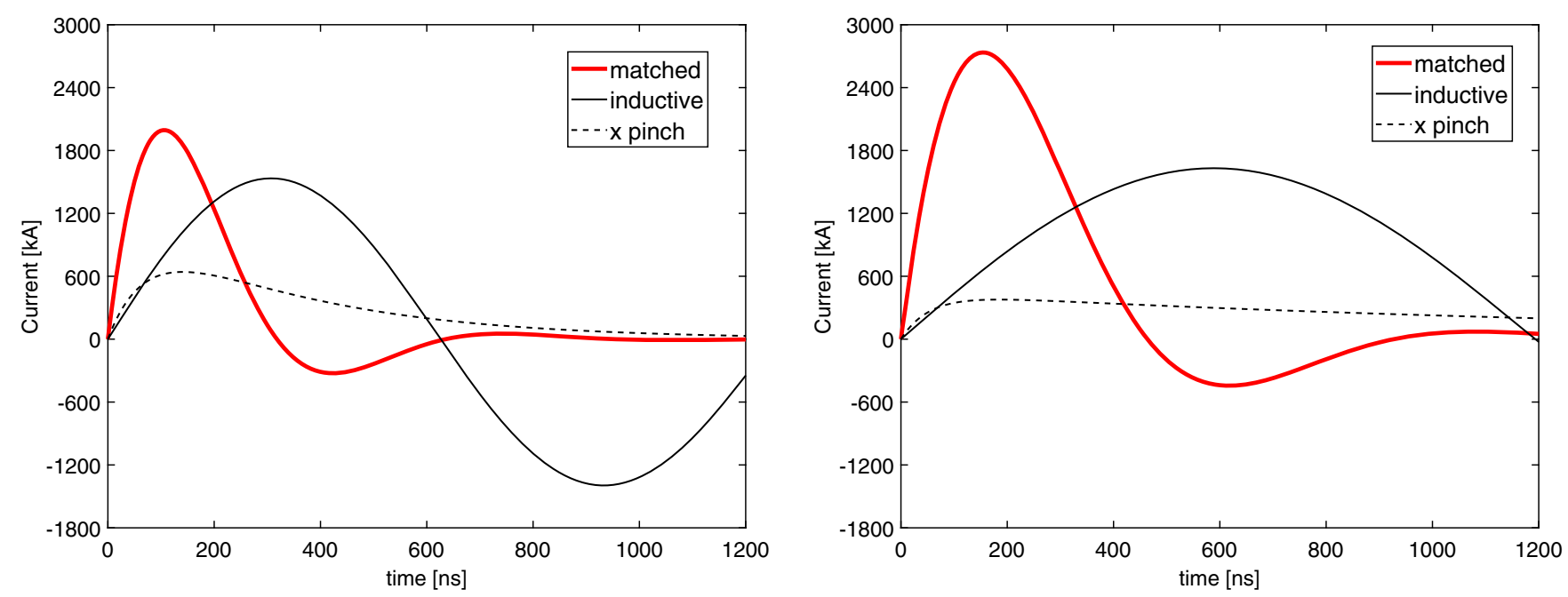

FIG. 9. Simulated output currents into matched, inductive, and x-pinch loads for a 40-brick standard pulser (left) and a 40-brick dry pulser (right). Both systems include a 0.3-nH TL.

circuits become highly overdamped, similarly to what was observed for the case with a 12-nH TL. The $d I / d t$ with the $\mathrm{x}$-pinch loads is $4.6 \mathrm{kA} / \mathrm{ns}$ for the standard pulser and $2.0 \mathrm{kA} / \mathrm{ns}$ for the dry pulser. Even though these values greatly exceed the 1-kA/ns threshold required for "good" $\mathrm{x}$-pinch radiation performance $[35,36]$ the use of a pulser comprised of a very large number of bricks is not very efficient, as the peak current is much smaller than what would be expected from a pulser of this size.

\section{Scaling the pulser's peak current with an $x$-pinch load}

For a pulser comprised of a small number of bricks (see Fig. 7), the $d I / d t$ is close to $1 \mathrm{kA} / \mathrm{ns}$, which, as a rule of thumb, is the minimum required $d I / d t$ for forming a highquality x-pinch radiation source [35]. With a large number

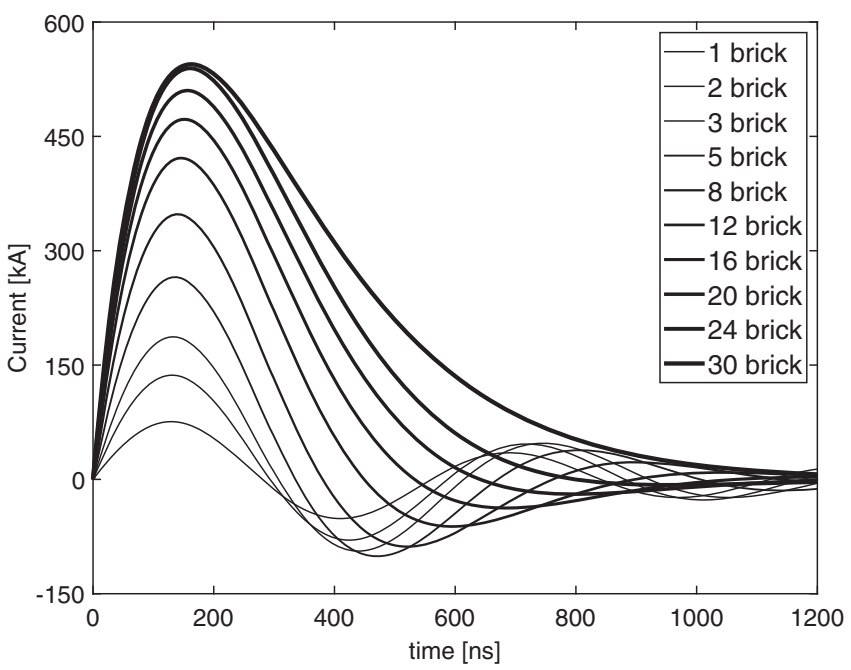

of bricks (see Figs. 8 and 9), the x-pinch load is largely overdamped, and the pulser's performance becomes inefficient. Thus, we might expect the optimal number of bricks for driving an $\mathrm{x}$-pinch load to lie somewhere in between $N=4$ and $N=40$. Simulation results for an x-pinch load driven by a pulser comprised of either 1, 2, 3, 5, 8, 12, 16, 20,24 , or 30 bricks (both for standard and dry brick configurations) are presented in Fig. 10. In all cases, the pulsers are comprised of bricks with $80-\mathrm{nF}, 100-\mathrm{kV}$ capacitors, the TLs are $6 \mathrm{nH}$, and the x-pinch load parameters are $10 \mathrm{nH}$ and $0.24 \Omega$.

With a small number of bricks, the analysis indicates that the current either exceeds the capacitor's maximum recommended current threshold (50 kA), or the $d I / d t$ drops below the minimum $d I / d t$ required for high-quality $\mathrm{x}$-pinch formation $(1 \mathrm{kA} / \mathrm{ns})$. For example, for a pulser

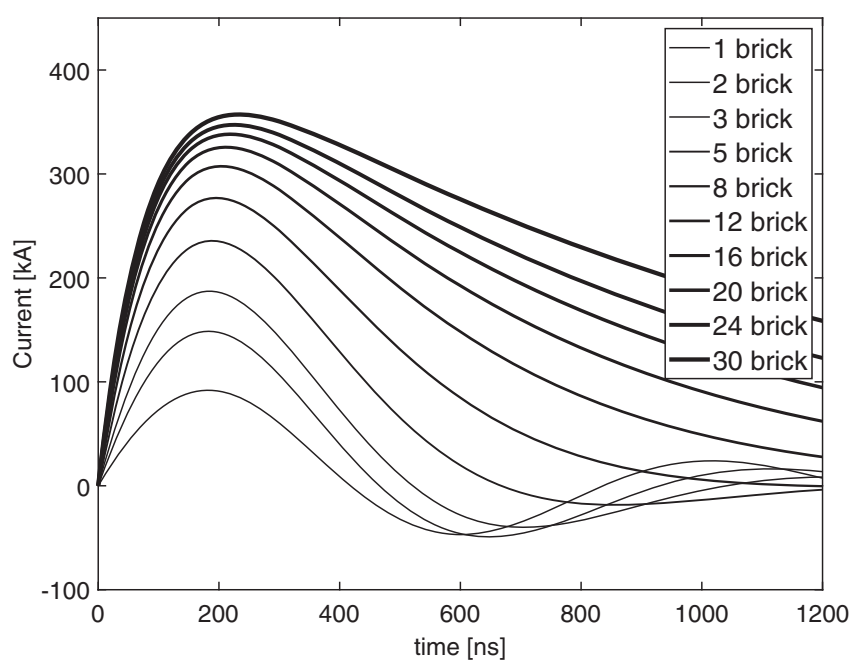

FIG. 10. Simulated output currents into an x-pinch load for a standard pulser (left) and a dry pulser (right), where the number of bricks is varied for each case. 
comprised of two standard bricks, the peak current into the $\mathrm{x}$-pinch load is $140 \mathrm{kA}$ and the corresponding $d I / d t$ is $1 \mathrm{kA} / \mathrm{ns}$. In this case, the charging voltage must be reduced to keep the current to less than $50 \mathrm{kA}$ per capacitor, but as a result, the corresponding $d I / d t$ drops below the $1-\mathrm{kA} / \mathrm{ns}$ $\mathrm{x}$-pinch threshold. For the pulser comprised of two dry bricks, the peak current is $150 \mathrm{kA}$ which is acceptable for this type of brick (the current is less than $50 \mathrm{kA}$ per capacitor), but the corresponding $d I / d t$ is only $0.8 \mathrm{kA} / \mathrm{ns}$. To simultaneously satisfy both requirements, the pulser must be comprised of at least three bricks, regardless of the type of brick used. Note that this conclusion is only true for bricks comprised of $80-\mathrm{nF}, 100-\mathrm{kV}$ capacitors and must be reevaluated for other capacitor values. For example, the Idaho State University (ISU) x-pinch pulser [20] is comprised of two dry bricks, but its capacitors are $140 \mathrm{nF}$ instead of $80 \mathrm{nF}$, which enables it to exceed the $1-\mathrm{kA} / \mathrm{ns}$ threshold and generate a high-quality $\mathrm{x}$-pinch radiation source (a single $\mathrm{x}$-ray burst from a few-micron spot size over a few-ns duration).

As we increase the total number of bricks beyond $N=20$, both the standard and dry-brick pulsers become very inefficient and their peak currents tend to saturate. For example, as we increase the number of bricks from 20 to 24 to 30 , peak currents increase from 510 to 540 to $545 \mathrm{kA}$ for the standard pulser and, correspondingly, from 340 to 350 to $360 \mathrm{kA}$ for the dry pulser. This is much less than might be expected from a pulser comprised of so many bricks.

It seems that the optimal number of bricks would be somewhere between 3 and 20 for both the standard and dry brick configurations: the lower limit is imposed by the requirements of providing at least $1 \mathrm{kA} / \mathrm{ns}$ into the $\mathrm{x}$-pinch load, and the upper limit represents approximately where the pulser's peak currents start to saturate. In general, for driving an x-pinch load with the resistance value assumed, a pulser comprised of standard bricks outperforms the drybrick pulser in both peak current and $d I / d t$.

\section{CONCLUSIONS AND DISCUSSION}

Throughout this paper, we have presented examples of how the standard-brick pulser compares to the dry-brick pulser assuming $80-\mathrm{nF}, 100-\mathrm{kV}$ capacitors for fixed brick parameters of $40 \mathrm{nF}$ and $160 \mathrm{nH}$ for the standardbrick pulser, and $160 \mathrm{nF}$ and $90 \mathrm{nH}$ for the dry-brick pulser. Here we further discuss how relations between the standard and dry-brick pulsers can be generalized for arbitrary brick parameters.

Considering Eqs. (2.4) and (3.4), when $L_{\text {extra }} \ll L_{\text {pulser }}$, the ratio between the peak currents for the standard- and dry-brick pulsers becomes

$$
\frac{I_{\text {system }}^{\text {dry }}}{I_{\text {system }}^{\text {stand }}}=\frac{\left[\left(0.55 V_{0} / \sqrt{L_{\text {brick }} / C_{\text {brick }}}\right) N\right]^{\text {dry }}}{\left[\left(0.55 V_{0} / \sqrt{L_{\text {brick }} / C_{\text {brick }}}\right) N\right]^{\text {stand }}} .
$$

Recalling that $C_{\text {brick }}^{\mathrm{dry}}=4 C_{\text {brick }}^{\text {stand }}$ and $V_{0}^{\text {stand }}=2 V_{0}^{\text {dry }}$, for pulsers comprised of the same number of bricks, $N$, it can be seen that (6.1) depends only on the corresponding brick inductances and is independent of the brick capacitances:

$$
I_{\text {system }}^{\mathrm{dry}} / I_{\mathrm{system}}^{\mathrm{stand}}=\sqrt{L_{\mathrm{brick}}^{\mathrm{stand}} / L_{\mathrm{brick}}^{\mathrm{dry}}} .
$$

For a 40-nF, 180-nH standard-brick pulser and a 160-nF, 90-nH dry-brick pulser, the ratio becomes $\sqrt{2}=1.41$, so the dry-brick pulser will generate a $40 \%$ higher peak current than the standard-brick pulser, as was evident from the earlier examples.

Considering Eqs. (2.4) and (4.6), when $L_{\text {extra }}>L_{\text {pulser }}$, and for a large $N$, the ratio between the peak currents for the standard and dry-brick pulsers becomes

$\frac{I_{\text {system }}^{\text {dry }}}{I_{\text {system }}^{\text {stand }}}=\frac{\left[\left(0.55 V_{0} / \sqrt{L_{\text {brick }} / C_{\text {brick }}}\right) \sqrt{L_{\text {brick }} / L_{\text {extra }}} \sqrt{N}\right]^{\text {dry }}}{\left[\left(0.55 V_{0} / \sqrt{L_{\text {brick }} / C_{\text {brick }}}\right) \sqrt{L_{\text {brick }} / L_{\text {extra }}} \sqrt{N}\right]^{\text {stand }}}$.

Again applying $C_{\text {brick }}^{\text {dry }}=4 C_{\text {brick }}^{\text {stand }}$ and $V_{0}^{\text {stand }}=2 V_{0}^{\text {dry }}$, as was done above for (6.2), and assuming that both pulsers having the same number of bricks $\left(N^{\text {stand }}=N^{\text {dry }}\right)$ and the same extra inductances $\left(L_{\text {extra }}^{\text {stand }}=L_{\text {extra }}^{\text {dry }}\right)$, it is evident that (6.3) is not only independent of the brick inductances, but is also independent of the brick capacitances-it is simply equal to one:

$$
I_{\text {system }}^{\mathrm{dry}} / I_{\text {system }}^{\text {stand }}=1 .
$$

This relation states that when $L_{\text {extra }}>L_{\text {pulser }}$, and for a large $N$, the peak current into the matched load is completely independent of the type of brick used, either standard or dry. We encountered this pulser behavior before, when we discussed Eq. (4.6) in Sec. IV for the specific brick parameters of $40 \mathrm{nF}$ and $160 \mathrm{nH}$ for the standard brick, and $160 \mathrm{nF}$ and $90 \mathrm{nH}$ for the dry brick. However, here, this statement can be seen more broadlythe peak current is not only independent of the type of brick but is also independent of the capacitance and inductance of the brick.

In summary, we have considered how two different brick topologies, standard and dry, affect a system's behavior. Considering matched loads at the single-brick level, relative to the bipolar-charged standard brick, the dry brick provides a larger output current from capacitors that have been unipolar charged. As we increase the total number of bricks, the scaling relations for the system output parameters depend on how large the extra inductance is (i.e., the combined inductance of the TL and the load) relative to the internal inductance of the pulser itself (i.e., the equivalent inductance of all the bricks connected in parallel). If the 
extra inductance is neglectable, then the output current is directly proportional to the number of bricks, and the drybrick pulser outperforms the standard-brick pulser [see Eq. (3.4)]. When the extra inductance cannot be neglected, and with a large number of bricks $N$, both the pulser's peak current and time to peak current scale as the $\sqrt{N}$ [see Eqs. (4.6) and (4.9)]. Interestingly, for large $N$ and nonnegligible $L_{\text {extra }}$, the current scaling coefficient is almost independent of the type of brick, while the time to peak current scaling coefficient varies significantly with the type of brick. How these scaling relations are affected by different loads has been further discussed in Sec. V.

For some applications, like the x-pinch load, the standard pulser may at first seem to be the better option, since it provides better (higher) $d I / d t$ performance. However, we note that the dry pulser is still a good alternative for the following reasons. First, the entire pulser can be operated without immersing its bricks in dielectric oil. Second, the dry brick can be thought of as an independent modular unit. That is, the dry brick has its own body, which fully encapsulates and insulates its components, whereas the standard brick is typically just an assembly of parts (two capacitors, a switch, electrodes, brackets, etc.), which must be mounted to a support structure in the pulser and submerged in dielectric oil for insulation. Consequently, the dry brick provides excellent flexibility in pulser assembly. An example is the Cinco accelerator [21], where the pulser's total current can be increased simply by adding more bricks to the parallel plate TL. Indeed, the dry brick's modularity and ease of operation are what make it a great choice for both large and small pulsed power applications.

\section{ACKNOWLEDGMENTS}

This work was supported in part by the NNSA Stewardship Sciences Academic Programs under DOE Cooperative Agreement No. DE-NA0003764, in part by the U.S. Office of Naval Research through the Young Investigator Program under Grant No. N00014-18-1-2499, and in part by the DOE Fusion Energy Sciences Program under Grants No. DE-SC0020239, No. DE-SC0016252, and the National Science Foundation under Grants No. PHY1725178 and No. PHY-1943939.

[1] B. M. Koval'chuk, V. A. Vizir', A. A. Kim, E. V. Kumpyak, S. V. Loginov, A. N. Bastrikov, V. V. Chervyakov, N. V. Tsoi, P. Monjaux, and D. Kh'yui, Fast primary storage device utilizing a linear pulse transformer, Russ. Phys. J. 40, 1142 (1997).

[2] A. A. Kim et al., Development and tests of fast 1-MA linear transformer driver stages, Phys. Rev. ST Accel. Beams 12, 050402 (2009).

[3] M. G. Mazarakis et al., High current 0.5-MA fast 100-ns linear transformer driver experiments, Phys. Rev. ST Accel. Beams 12, 050401 (2009).
[4] R. M. Gilgenbach et al., MAIZE: A 1 MA LTD-driven Z-pinch at The University of Michigan, AIP Conf. Proc. 1088, 259 (2009).

[5] M. G. Mazarakis et al., High-current linear transformer driver development at Sandia National Laboratories, IEEE Trans. Plasma Sci. 38, 704 (2010).

[6] J. R. Woodworth, W. E. Fowler, B. S. Stoltzfus, W. A. Stygar, M. E. Sceiford, M. G. Mazarakis, H. D. Anderson, M. J. Harden, J. R. Blickem, R. White, and A. A. Kim, Compact $810 \mathrm{kA}$ linear transformer driver cavity, Phys. Rev. ST Accel. Beams 14, 040401 (2011).

[7] A. A. Kim, M. G. Mazarakis, V. A. Sinebryukhov, S. N. Volkov, S. S. Kondratiev, V. M. Alexeenko, F. Bayol, G. Demol, and W. A. Stygar, Square pulse linear transformer driver, Phys. Rev. ST Accel. Beams 15, 040401 (2012).

[8] T. Liang, F. Sun, X. Jiang, Z. Zhang, J. Yin, Z. Wang, and A. Qiu, Design of the $500 \mathrm{kA}$ linear transformer driver stage, in Proceedings of the 4th International Particle Accelerator Conference, IPAC-2013, Shanghai, China, 2013 (JACoW, Shanghai, China, 2013), https://accelconf .web.cern.ch/IPAC2013/papers/mopwa017.pdf.

[9] C. Liang, L. Zhou, F. Sun, J. Zeng, M. Li, Z. Wang, Z. Li, and $X$. Peng, A repetitive $800 \mathrm{kA}$ linear transformer drivers stage for Z-pinch driven fusion-fission hybrid reactor, Laser Part. Beams 33, 535 (2015).

[10] Z. Lin, Z. Li, Z. Wang, C. Liang, M. Li, J. Qi, and Y. Chu, Design of a 5-MA 100-ns linear-transformer-driver accelerator for wire array Z-pinch experiments, Phys. Rev. Accel. Beams 19, 030401 (2016).

[11] P.-A. Gourdain, M. Evans, B. Foy, D. Mager, R. McBride, and R. Spielman, HADES: A high amperage driver for extreme states, arXiv:1705.04411.

[12] B. M. Kovalchuk, A. V. Kharlov, V. B. Zorin, and A. A. Zherlitsyn, A compact submicrosecond, high current generator, Rev. Sci. Instrum. 80, 083504 (2009).

[13] A. A. Zherlitsyn, B. M. Kovalchuk, and G. V. Smorudov, Capacitor units with air insulation for linear transformers, Instrum. Exp. Tech. 52, 802 (2009).

[14] B. M. Kovalchuk, A. V. Kharlov, E. V. Kumpyak, G. V. Smorudov, and A. A. Zherlitsyn, Capacitor blocks for linear transformer driver stages, Rev. Sci. Instrum. 85, 013501 (2014).

[15] A. A. Kim, B. M. Kovalchuk, A. N. Bastrikov, V. G. Durakov, S. N. Volkov, and V. A. Sinebryukhov, $100 \mathrm{~ns}$ current rise time LTD stage, in PPPS-2001 Pulsed Power Plasma Science 2001. 28th IEEE International Conference on Plasma Science and 13th IEEE International Pulsed Power Conference, Las Vegas, NV, 2002, https://doi.org/ 10.1109/PPPS.2001.1001840.

[16] B. M. Kovalchuk, A. A. Kim, E. V. Kumpjak, N. V. Zoi, J. P. Corley, Y. W. Struve, and D. L. Johnson, Multigap switch for Marx generators, in PPPS-2001 Pulsed Power Plasma Science 2001. 28th IEEE International Conference on Plasma Science and 13th IEEE International Pulsed Power Conference, Las Vegas, NV, USA, 2002, https:// doi.org/10.1109/PPPS.2001.1001907.

[17] A. A. Kim, V. G. Durakov, S. N. Volkov, A. N. Bastrikov, B. M. Kovalchuk, V. A. Sinebryukhov, S. V. Frolov, V. M. Alexeenko, L. Véron, M. Toury, C. Vermare, R. Nicolas, F. Bayol, and C. Drouilly, Gas switch performance depending 
on current in the circuit, in Proceedings of the 14th International Symposium High-Current Electronics, Tomsk, 2006 (HCEI, Tomsk, 2006), https://www.hcei.tsc .ru/conf/2010/cat/proc_2006/shce/Paper_074.pdf.

[18] B. M. Kovalchuk, A. V. Kharlov, E. V. Kumpyak, and A. A. Zherlitsyn, Pulse generators based on air-insulated linear-transformer-driver stages, Phys. Rev. ST Accel. Beams 16, 050401 (2013).

[19] S. N. Bland, K. H. Kwek, S. J. P. Stafford, J. B. R. Winters, D. Hinshelwood, and R. B. Spielman, A new pulsed power facility for isentropic compression experiments, at the APS Shock Compression of Condensed Matter, 2013, https:// doi.org/10.1109/PLASMA.2013.6633488.

[20] R. V. Shapovalov, R. B. Spielman, and G. R. Imel, An oilfree compact X-pinch plasma radiation source: Design and radiation performance, Rev. Sci. Instrum. 88, 063504 (2017).

[21] T. E. Bejines, R. B. Spielman, and D. B. Reisman, Cinco: A compact high-current driver for high-energy-density physics, in 2019 IEEE Pulsed Power \& Plasma Science (PPPS) (Orlando, FL, 2020), 10.1109/PPPS34859.2019 .9009668 .

[22] A. A. Kim, B. M. Kovalchuk, V. A. Kokshenev, A. V. Shishlov, N. A. Ratakhin, V. I. Oreshkin, V. V. Rostov, V. I. Koshelev, and V. F. Losev, Review of high-power pulsed systems at the Institute of High Current Electronics, Matter Radiat. Extremes 1, 201 (2016), https://doi.org/ 10.1016/j.mre.2016.08.001.

[23] A. A. Kim and M. G. Mazarakis, The story of the LTD development, IEEE Trans. Plasma Sci. 48, 749 (2020).

[24] R. Shapovalov, M. Adams, M. Evans, H. Hasson, J. Young, I. West-Abdallah, and P-A. Gourdain, Low-inductance load test of a new 250-kA, 150-ns pulser for fast x-pinch sources, in 2019 IEEE Pulsed Power \& Plasma Science, Orlando, FL, 2020, https://doi.org/10.1109/PPPS34859 .2019.9009748.

[25] M. G. Mazarakis and R. B. Spielman, A compact, highvoltage e-beam pulser, in 12th IEEE International Pulsed Power Conference (Monterey, CA, 1999), https://doi.org/ 10.1109/PPC.1999.825498.

[26] J. R. Woodworth, J. A. Alexander, F. R. Gruner, W. A. Stygar, M. J. Harden, J. R. Blickem, G. J. Dension, F. E. White, L. M. Lucero, H. D. Anderson, L. F. Bennett, and S. F. Glover, D. Van DeValde, and M. G. Mazarakis, Low-inductance gas switches for linear transformer drivers, Phys. Rev. ST Accel. Beams 12, 060401 (2009).
[27] J. R. Woodworth, W. A. Stygar, L. F. Bennett, M. G. Mazarakis, H. D. Anderson, M. J. Harden, J. R. Blickem, F. R. Gruner, and R. White, New low inductance gas switches for linear transformer drivers, Phys. Rev. ST Accel. Beams 13, 080401 (2010).

[28] R. D. McBride et al., A primer on pulsed power and linear transformer drivers for high energy density physics applications, IEEE Trans. Plasma Sci., 46, 3928 (2018).

[29] S. Braginskii, Theory of the development of a spark channel, Zh. Eksp. Teor. Fiz. 34, 1548 (1958), http:// www.jetp.ac.ru/cgi-bin/dn/e_007_06_1068.pdf.

[30] M. L. Kiefer and M. M. Widner, SCREAMER-A singleline pulsed-power design tool, in IEEE Pulsed Power Conference, Washington, DC, 1985, edited by P. J. Turchi and M.F. Rose (IEEE, Piscataway, NJ, 1985), p. 685 , https://inis.iaea.org/search/search.aspx?orig_q= $\mathrm{RN}: 18080265$.

[31] T. H. Martin, J. F. Seamen, and D. O. Jobe, Energy losses in switches, in 9th IEEE International Pulsed Power Conference, Albuquerque, NM, 1993, https://doi.org/ 10.1109/PPC.1993.513375.

[32] D. B. Reisman et al., Pulsed power accelerator for material physics experiments, Phys. Rev. ST Accel. Beams 18, 090401 (2015)..

[33] R. B. Spielman, M. L. Kiefer, K. L. Shaw, K. W. Struve, and M. M. Winde, SCREAMER: A pulsed Power Design Tool: User's Guide for Version 4.3.3, 2019 (unpublished), https://www.isu.edu/iac/research/screamer/.

[34] R. B. Spielman and Y. Gryazin, SCREAMER: An optimized pulsed-power circuit-analysis tool, in IEEE International Power Modulator and High Voltage Conference, San Francisco, CA, 2017, https://doi.org/10.1109/IPMHVC .2016 .8012884 .

[35] T. A. Shelkovenko, S. A. Pikuz, J. D. Douglass, R. D. McBride, J. B. Greenly, and D. A. Hammer, Multiwire X-pinches at 1-MA current on the COBRA pulsedpower generator, IEEE Trans. Plasma Sci. 34, 2336 (2006).

[36] G. A. Mesyats et al., X-pinch source of subnanosecond soft $\mathrm{X}$-ray pulses based on small-sized low-inductance current generator, J. Exp. Theor. Phys. 111, 363 (2010).

Correction: NSF grant numbers were missing in the Acknowledgments section and have been inserted. 